This document is the unedited Author's version of a Submitted Work that was subsequently accepted for publication in ACS Applied Materials and Interfaces, copyright (c) American Chemical Society after peer review. To access the final edited and published work see:

https://dx.doi.org/10.1021/acsami.9b23487. 


\section{Chitin Nanofiber Paper towards Optical (Bio)sensing Applications}

Tina Naghdi ${ }^{\dagger, \ddagger}$, Hamed Golmohammadi ${ }^{\dagger},{ }^{*}$, Hossein Yousefi ${ }^{\S}$, Mohammad Hosseinifard ${ }^{\dagger}$ Uliana Kostiv, $\perp$ Daniel Horák, ${ }^{\perp}$ and Arben Merkoçi ${ }^{*}$, ,

† Nanosensor Bioplatforms Laboratory, Chemistry and Chemical Engineering Research Center of Iran, 14335-186, Tehran, Iran,

‡ ICN2 - Nanobioelectronics \& Biosensors Group, Institut Catala de Nanociencia i Nanotecnologia, Campus UAB, Bellaterra, Barcelona 08193, Spain,

$\S$ Laboratory of Sustainable Nanomaterials, Department of Wood Engineering and Technology, Gorgan University of Agricultural Sciences and Natural Resources, Gorgan 4913815739, Iran,

$\perp$ Institute of Macromolecular Chemistry, Academy of Sciences of the Czech Republic, Heyrovského Sq. 2, Prague 6162 06, Czech Republic,

॥ ICREA, Pg. Lluís Companys 23, 08010 Barcelona, Spain.

* Corresponding authors: Hamed Golmohammadi, Arben Merkoçi

Email : golmohammadi@ccerci.ac.ir

arben.merkoci@icn2.cat

\section{ABSTRACT}

Because of numerous inherent and unrivalled features of nanofibers made of chitin, the second most plentiful natural-based polymer (after cellulose), including affordability, abundant nature, biodegradability, biocompatibility, commercial availability, flexibility, transparency and extraordinary mechanical and physicochemical properties, chitin nanofibers (ChNFs) are being applied as one of the most appealing bionanomaterials in a myriad of fields. Herein, we exploited the beneficial properties offered by ChNF paper to fabricate transparent, efficient, biocompatible, flexible and miniaturized optical sensing bioplatforms via embedding/immobilizing various plasmonic nanoparticles (silver and gold 
nanoparticles), photoluminescent nanoparticles (CdTe quantum dots, carbon dots and $\mathrm{NaYF}_{4}: \mathrm{Yb}^{3+} @ \mathrm{Er}^{3+} \& \mathrm{SiO}_{2}$ upconversion nanoparticles) along with colorimetric reagents (curcumin, dithizone and etc.,) in 3D nanonetwork scaffold of ChNF paper. Several configurations, including 2D multi-wall, 2D cuvette patterns with hydrophobic barriers/walls and hydrophilic test zones/channels were easily printed using laser printing technology or punched as spot patterns on the dried ChNF paper-based nanocomposites to fabricate the (bio)sensing platforms. A variety of (bio)chemicals as model analytes were used to confirm the efficiency and applicability of the fabricated ChNF paper-based sensing bioplatforms. The developed (bio)sensors were also coupled with smartphone technology to take the advantages of smartphone-based monitoring/sensing devices along with the Internet of Nano Things (IoNT)/the Internet of Medical Things (IOMT) concepts for easy-to-use sensing applications. Building upon the unrivaled and inherent features of ChNF as a very promising bionanomaterial, we foresee that the ChNF paper-based sensing bioplatforms will emerge new opportunities for the development of innovative strategies to fabricate cost-effective, simple, smart, transparent, biodegradable, miniaturized, flexible, portable, and easy-to-use (bio)sensing/monitoring devices.

KEYWORDS: Chitin nanofibers; Optical sensor; Sensing bioplatform; Bionanocomposite, Internet of Things.

\section{INTRODUCTION}

Despite the tremendous and profound impact of using polymeric materials in everyday life, the growing concerns about the use of these materials due to adverse effects associated with their usage on human and environmental health have 
attracted enormous attention during the recent decades to replace them with naturalbased biopolymers ${ }^{1-2}$. The ever-increasing demand to natural-based biopolymers is attributed to their inherent non-toxicity, renewability, and biodegradability properties 1-3. Among the bio-based polymers, chitin as the second most plentiful natural-based polymer (after cellulose) and the most abundant natural amino-polysaccharide with an annual biosynthesis around $10^{10-10^{11}}$ tons, which is made of $\beta-(1,4)-2$ acetamido-2-deoxy-D-glucose and mainly found in the exoskeletons of numerous organisms including marine shrimp and crabs, insects, fungi and yeasts, has received considerable attention in several areas on account of its inherent and beneficial features such as low cost, abundant nature, non-toxicity, renewability, biodegradability, sustainability and biocompatibility compared to conventional polymeric materials ${ }^{4-16}$. This biopolymer is composed of chitin nanofibrils or chitin nanofibers (ChNFs) at the nanoscale with the diameter ranging from 3 to $40 \mathrm{~nm}$ and length longer than 5 micrometers. Interestingly, ChNFs as one of the natural-based nanomaterials, which are completely produced from chitin compounds through topdown approaches including chemical, mechanical or chemi-mechanical routes, not only have all the aforementioned beneficial properties of chitin but bring optical transparency and high reinforcing capability as well. Furthermore, because of fascinating features including high aspect ratio, strong hydrogen bonding, extended crystalline structures and other remarkably unique characteristics of nanomaterials along with the aforementioned properties of chitin, ChNFs are being exploited as one of the most desirable bionanomaterials in various fields including food, pharmaceutical, biomedical, cosmetic industries, energy, electronics, electrical and optical devices ${ }^{4-18}$. Special examples of myriad applications of ChNFs include the low-calorie foods ${ }^{19}$, natural biodegradable films to preserve foods from microbial 
deterioration ${ }^{20}$, filters for purification of water ${ }^{21}$, medical devices and wound-healing dressings ${ }^{22-23}$, drug delivery ${ }^{8}$, organic light-emitting diodes (OLED) ${ }^{7}$, thin film flexible transistors and solar cells ${ }^{7}$, tissue engineering applications ${ }^{17}$, energy storage devices ${ }^{9}$, and novel aerogels ${ }^{24}$. Nevertheless, despite the diverse uses of ChNFs, so far, they have been rarely exploited for optical (bio)sensing applications. On the other hand, although paper-based analytical devices as efficient sensing platforms have recently received great attention for (bio)sensing applications ${ }^{25-30}$ because of the advantageous properties of paper including affordability, abundant availability, versatility, biodegradability, printability, flexibility, portability, and porosity, however, there are major hurdles restricting their development. Namely, the intrinsic features of paper including large surface roughness, low stability and mechanical strength in the aqueous media, and especially its optical opaqueness can hinder the further progress of paper-based analytical devices in optical sensing applications ${ }^{3,}$ ${ }^{31}$. Accordingly, it is urgent to develop new optical sensing bioplatforms that, while having the beneficial properties of paper-based sensors, are optically transparent and possess more chemical, mechanical, and thermal stability as well.

We have already fabricated optical sensing platforms using bacterial cellulose (BC) nanopaper ${ }^{31-34}$. In the current research, the optical sensing platforms were fabricated from ChNF paper. Unlike BC nanopaper, ChNFs are isolated from very cheap raw material of shrimp's shells, which are available in abundant amount almost in every country. The production of BC nanopaper possesses timeconsuming and costly process as well, while that of ChNF is inexpensive, easy and fast. Moreover, unlike BC nanopaper, owing to the presence of the amide and hydroxyl reactive functional groups on the surface of ChNF paper, there is no need to modify the ChNF papers through chemical reactions for immobilization of different 
(bio)chemicals/nanomaterials in their nanoscaffold. On the other hand, the conversion of very cheap and abundant raw materials of shrimp's shells into ChNFs and their application in the fabrication of sensing platforms can be of great importance from technical, economic and environmental points of view.

Herein, we exploited the beneficial features offered by ChNF paper to fabricate transparent, flexible, biocompatible, lightweight and efficient optical sensing bioplatforms through embedding various plasmonic nanoparticles (NPs): silver and gold NPs; photoluminescent NPs (PLNPs): N-Acetyl L-Cysteine/TGA capped cadmium telluride (CdTe) quantum dots (QDs), carbon dots (CDs) and $\mathrm{NaYF}_{4}: \mathrm{Yb}^{3+} @ \mathrm{Er}^{3+} \& \mathrm{SiO}_{2}$ up-conversion nanoparticles (UCNPs); along with colorimetric reagents (curcumin, dithizone and etc.,) in 3D nanonetwork scaffold of ChNF paper. To fabricate the ChNF paper-based (bio)sensing platforms, several patterns including 2D multi-wall and 2D cuvette patterns with hydrophobic walls and hydrophilic test zones were easily printed by using an office laser printer or punched as spot patterns on the dried films of the fabricated ChNF paper-based nanocomposites.

A various of (bio)chemicals were tested as model analytes to confirm the efficiency of the developed ChNF paper-based sensing bioplatforms.

The fabricated ChNF paper-based sensing bioplatforms were also coupled with smartphone technology for (bio)sensing applications. Additionally, we have designed an Internet of Nano Things (loNT)/ Internet of Medical Things (loMT) Model to improve potential applicability and efficiency of the developed smartphone-based (bio)sensing device via loT concept. Proposed by Ashton in $1999^{35}$, loT has revolutionized our world in so many different ways and offered new solutions for our 
complex world. IoT is a combination of data from sensors and networks that provide different smart services ${ }^{36}$. It links all kinds of connected "Things" into a comprehensive network of interrelated computing intelligence without the involvement of a human ${ }^{37}$. It aims to add computer-based logic to plenty of things which can then be monitored or controlled by analytics or machines ${ }^{38}$. Besides, loT ecosystem contains web-enabled smart devices that use implanted processors, sensors and communication hardware to collect, send and use the data they acquire from their settings ${ }^{36}$. Nowadays, loT is known as a "megatrend" which goes beyond the limitations of all scientific and engineering disciplines and institutes an integrating technical evolution to improve our life ${ }^{39}$. Moreover, inter-connection of Nano-sensors and Nano-devices with Internet has also led to a new evolution called "Internet of Nano Things" (IoNT) ${ }^{40-41}$. The coupling of ChNF paper-based sensors to smartphones could significantly increase the potential applications of these sensing bioplatforms and open up new opportunities to develop affordable, portable, and easy-to-use sensing/monitoring devices, especially in the developing smart cities.

\section{RESULTS AND DISCUSSION}

The aim of the current study is the fabrication of transparent, flexible, biocompatible, compact, portable, and efficient ChNF paper-based optical sensing bioplatforms through immobilization/embedding of various plasmonic NPs, PLNPs and colorimetric reagents in 3D nanoscaffold of ChNF paper. In fact, in this work, we are intending to exploit unrivaled and beneficial features offered by ChNF paper in combination with fascinating and unique photophysical properties of plasmonic and PLNPs and colorimetric chemicals to develop bionanocomposites that can be utilized as novel (bio)sensing platforms, which are compatible with the World Health 
Organization's ASSURED (affordable, sensitive, specific, user-friendly, rapid \& robust, equipment free and deliverable to the end-users) criteria for the ideal diagnostic devices/assays, for easy (bio)chemical sensing applications.

The ChNF paper used throughout this work, as a thin sheet fully made from ChNFs, was produced from shells of shrimp (Littopenaeus vannamei) through a top-down chemi-mechanical approach. The experimental steps to produce ChNF paper are shown in Figure S1. The fibers diameter of the produced ChNF paper was in the range of $5-55 \mathrm{~nm}$ (average: $26 \pm 8 \mathrm{~nm}$ ). The crystallinity index, tensile strength, Young's modulus and strain-at-break of the produced ChNF paper were 89\%, 135 $\mathrm{MPa}, 7.7 \mathrm{GPa}$, and $8.2 \%$, respectively (See the Experimental section and Figures S2-S4 in the Supporting Information for more details on the production and characterization of the ChNF paper). The high mechanical properties of ChNF paper are attributed to the high degree of crystallinity, high aspect ratio, and specific surface area as well as its network structure.

In this section, the preparation of different ChNF paper-based nanocomposites, focusing on their fabrication, mechanism of sensing and also characterization via UV-visible spectroscopy (UV-vis), field emission scanning electron microscopy (FESEM), energy-dispersive X-ray spectroscopy (EDX), and Thermogravimetric analysis (TGA) techniques will be discussed. Various patterns with hydrophilic test zones/channels and hydrophobic barriers/walls were created on the fabricated ChNF paper-based sensing bioplatforms using laser printing technology. 


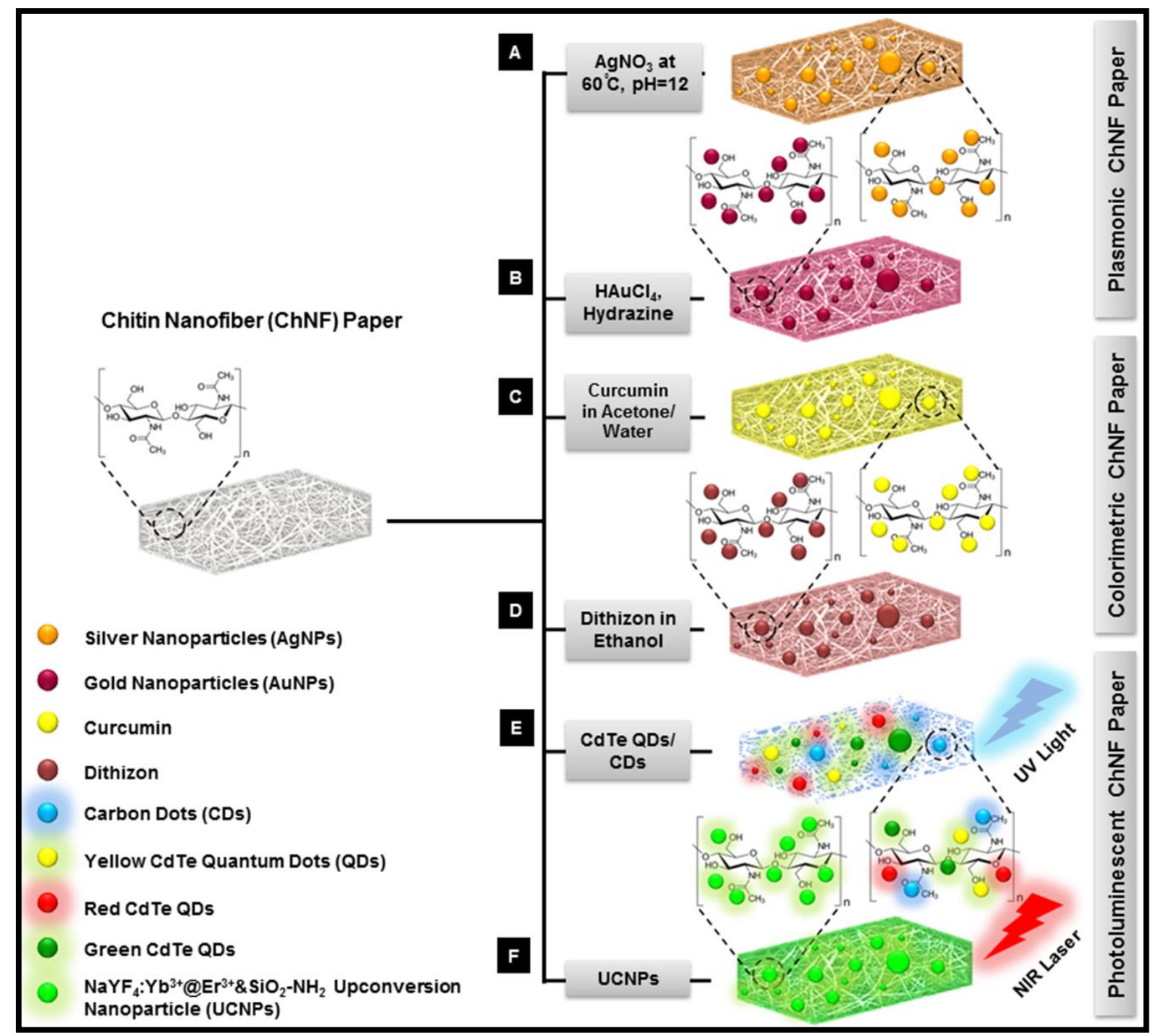

Figure 1. Schematic representation for the fabrication of different chitin nanofiber (ChNF) paper-based nanocomposites. (A and B) Fabrication of plasmonic ChNF paper-based nanocomposites: (A) silver nanoparticles-ChNF paper (AgNPs-ChNF paper); (B) gold nanoparticles ChNF paper (AuNPs-ChNF paper). (C and D) Fabrication of colorimetric ChNF paper-based nanocomposites: (C) Curcumin-ChNF paper (Cur- ChNF paper); (D) Dithizon-ChNF paper (DTZ- ChNF paper). (E and F) Fabrication of photoluminescent ChNF paper-based nanocomposites: (E) CdTe@ZnS quantum dots ChNF paper (QDs- ChNF paper), and Carbon dots- ChNF paper (CDsChNF paper); (F) aminosilica-coated $\mathrm{NaYF}_{4}: \mathrm{Yb}^{3+} @ \mathrm{Er}^{3+}$ upconversion NPs-ChNF paper (UCNPs- ChNF paper). 
Several strategies have been employed to embed and immobilization of different reagents in ChNF paper to fabricate various ChNF paper-based nanocomposites, which are schematically illustrated in Figure 1, including in situ synthesis of metallic NPs in ChNF paper through in situ chemical reduction of noble metal ions to metallic NPs by using the hydroxyl and amide functional groups of the ChNFs as reducing agents (Pathway A; Figure 1A); by first immobilization of noble metal ions within the ChNF paper substrate and subsequently chemical reduction of noble metal ions to metallic NPs after addition of chemical reducing agents (Pathway B; Figure 1B); by in situ embedding/immobilization of hydrophobic chemical reagents into the fibril network of the ChNF paper via hydrophilic-hydrophobic interactions (Pathway C,D; Figure 1C,D); and by direct coupling the functionalized NPs with functional groups of the ChNF paper (Pathway E,F; Figure 1E,F). The plasmonic ChNF paper-based nanocomposites (AgNPs-ChNF paper and AuNPs-ChNF paper) were fabricated via Pathways $A$ and $B$, respectively. The colorimetric ChNF paper-based nanocomposites (Curcumin/ChNF paper (Cur-ChNF paper) and Dithizone/ChNF paper (DTZ-ChNF paper) were fabricated via Pathways $C$ and D; respectively. The photoluminescent ChNF paper-based nanocomposites (QDs-ChNF papers, CDsChNF paper and UCNP-ChNF papers) were also fabricated via Pathways $E$ and $F$, respectively.

To fabricate the (bio)sensing platforms, various patterns with desired designs/shapes, including 2D multi-wall and 2D cuvette patterns with hydrophobic barriers/walls and hydrophilic test zones/channels can be easily created by using an office laser printer via direct-printing the hydrophobic toner layer on the fabricated ChNF paper-based nanocomposites (see Figures $2 \mathrm{~A}$ and $2 \mathrm{~B}$ ). The dried films of the fabricated ChNF paper-based nanocomposites can also be easily cut using a hole 
punch to fabricate spot patterns for individual assays (See Figure 2C). The flexibility and the created hydrophobic (black parts) and hydrophilic (colorless parts) areas on the fabricated ChNF paper-based sensing platforms have also been depicted in Figure $2 \mathrm{D}$, and $2 \mathrm{E}$, respectively.

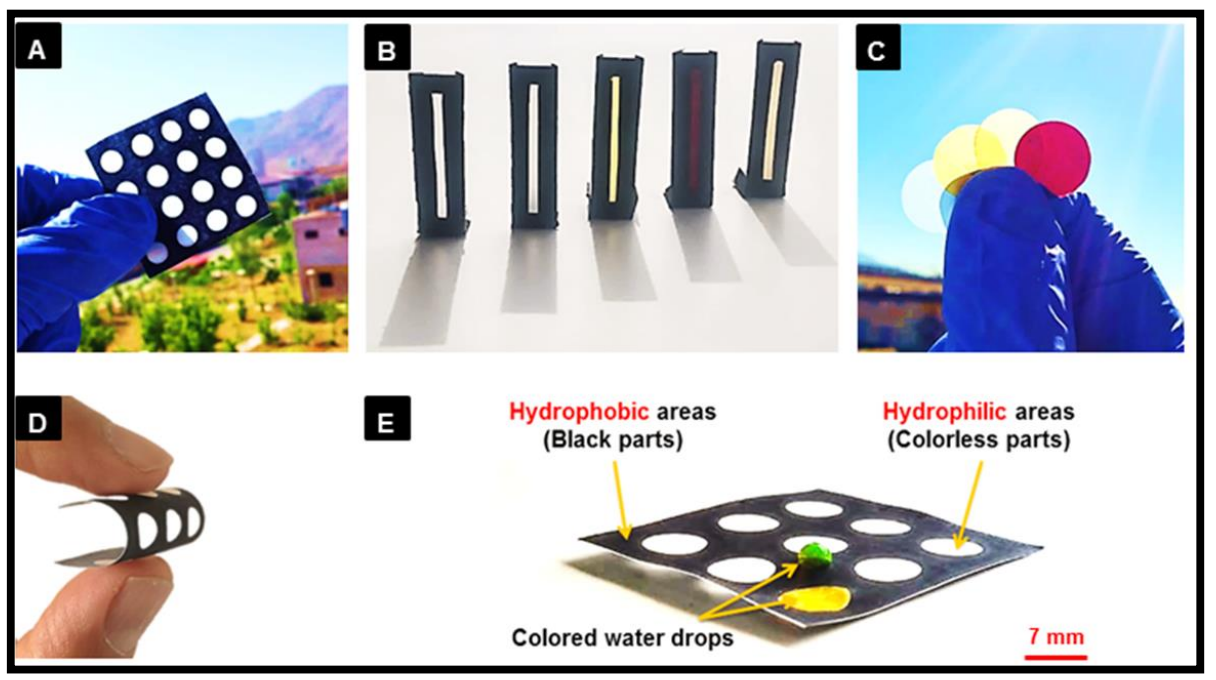

Figure 2. Pictures of the fabricated ChNF paper-based sensing platforms with (A) 2D multi-wall, (B) 2D cuvette and (C) spot patterns. (D) A picture showing the flexibility of the fabricated ChNF paper-based sensing platform. (E) A picture of the fabricated ChNF paper-based sensing platform: comparison of hydrophobic (black parts) and hydrophilic (colorless parts) areas after applying a drop (10 $\mu \mathrm{L})$ of colored water.

To verify the applicability of the developed ChNF paper-based sensing bioplatforms, they were exploited for optical sensing of various (bio)chemicals.

Interestingly, owing to optical transparency of the developed ChNF paper-based sensing bioplatforms, their color changes (e.g., fluorescence or absorbance intensity) upon the addition of analytes can be monitored spectroscopically using a spectrofluorometer/spectrophotometer or visually by a naked eye. 
To enhance the applicability of the developed ChNF paper-based sensing bioplatforms, they were also coupled with smartphone technology as a new generation of easy-to-use, cost-effective, rapid, and portable monitoring devices. Full details on all experimental procedures and setups are presented in the Experimental section and Supporting Information.

In the present work, the photoluminescence and UV-vis absorption spectra of the fabricated ChNF-based optical sensing platforms were carried out using a spectrofluorometer/spectrophotometer. The images, color intensities, and corresponding determined concentrations of the test zones of the fabricated ChNF paper-based optical sensing platforms were taken and processed using the developed smartphone-based 3D-printed platform/setup.

\subsection{Plasmonic ChNF paper-based optical sensing platforms}

In this work, plasmonic sensors were made using two types of noble metals ( $\mathrm{Ag}$ and $\mathrm{Au}$ ), which were synthesized/loaded into the ChNF paper. Since the color changes of noble metallic NPs, which associated with their surface plasmon absorption bands, are extremely dependent on their inter-particle spacing, composition, shape, and size, as well as the dielectric properties of their surrounding medium, they have been extensively employed as versatile and efficient platforms in sensing applications ${ }^{42-45}$. One of the beneficial ways that strongly affects the characteristics of plasmonic NPsbased sensors is their immobilization/synthesis on appropriate substrates ${ }^{33}$. For this purpose, we firstly in situ synthesized AgNPs in the ChNF paper. For the synthesis of AuNPs in the ChNF paper, we used two methods: I) in situ synthesis of AuNPs within the ChNF paper as a reducing agent and II) synthesis of AuNPs using 
hydrazine as a reducing agent and then embedding the synthesized AuNPs within the scaffold of ChNF paper. The images and scanning electron micrographs of bare ChNF paper, the fabricated AgNPs-ChNF paper and the fabricated AuNPs-ChNF paper (using hydrazine) are shown in Figure 3A-C, respectively. The cross-section scanning electron micrographs and phase image of bare ChNF paper, the fabricated AgNPs-ChNF paper and the fabricated AuNPs-ChNF paper (using hydrazine) are also shown in Figure S5A-C and S5D-F, respectively, which confirm the homogeneous distribution of the plasmonic NPs inside the ChNF paper. The image, UV-vis spectrum, and scanning electron micrograph of the in situ synthesized AuNPs-ChNF paper are shown in Figure S6. EDX spectra and TGA analysis were also carried out to verify the synthesis of AgNPs and AuNPs in the ChNF paper matrix (see Supporting Information Figure S7).

We then used the fabricated AgNPs-ChNF paper as plasmonic sensors for optical sensing of the drug methimazole (MTZ) and the toxic compound cyanide $\left(\mathrm{CN}^{-}\right)^{32-33}$. We observed the color changes of the AgNPs-ChNF papers from yellow to dark yellow by increasing the MTZ concentration, along with a red-shift to longer wavelengths in the UV-vis absorption spectra (see Figure 3D) and an increase in the size of AgNPs due to aggregation of AgNPs in the AgNPs-ChNF paper (see Figure 3B-b, Supporting Information Figure S8D). A color change from yellow toward light yellow is also observed by increasing the concentration of $\mathrm{CN}^{-}$, which is accompanied with a shift to shorter wavelengths (blue-shift) in the UV-vis absorption spectra (see Figure 3E) and a decrease in the size of AgNPs owing to the etching of AgNPs in the AgNPs-ChNF paper (see Figure 3B-c). Monitoring of MTZ and $\mathrm{CN}^{-}$is based on the high affinity of the $\mathrm{CN}^{-}$ions and thiol groups of MTZ to the AgNPs- 
ChNF paper, which consequently leads to changes in the size-dependent optical properties, surface plasmon absorption bands, and color of the AgNPs-ChNF paper.

To demonstrate the potential of the fabricated AuNPs-ChNF paper as a plasmonic sensor, it was employed for optical sensing of the noxious and harmful heavy metal ion $\mathrm{Hg}^{2+}$. Figure 3F illustrates the color changes of the AuNPs-ChNF papers from red to purple, along with a red-shift in their UV-vis absorbance spectra upon addition of $\mathrm{Hg}^{2+}$. The respective FE-SEM micrograph, which shows an increase in the size of AuNPs in the presence of $\mathrm{Hg}^{2+}$ due to the aggregation of AuNPs in the AuNPs-ChNF paper, is also illustrated in Figure 3C-b. The calibration curves and analytical characteristics corresponding to these experiments (the AgNPs-ChNF papers for MTZ and $\mathrm{CN}^{-}$sensing and the AuNPs-ChNF paper for $\mathrm{Hg}^{2+}$ sensing) are presented in Supporting Information Figure S8 A-C and Table S1. The size-distribution of the fabricated plasmonic ChNF papers were roughly estimated by image processing from FE-SEM images and the results confirmed the recommended size-depending sensing mechanisms (see Supporting Information Figure S8D and E). To evaluate the selectivity of the fabricated plasmonic ChNF paper-based optical sensing platforms, the competitive experiments were independently performed at a fixed concentration of $\mathrm{CN}^{-}(3 \mathrm{ppm})$ and MTZ (100 ppm) for AgNPs-ChNF paper, and $\mathrm{Hg}^{2+}$ (10 ppm) for AuNPs-ChNF paper in the presence of some chemicals probably existing in water samples. Figure S9-11, indicate the good selectivity of the developed plasmonic ChNF paper-based optical sensing platforms toward $\mathrm{CN}^{-}, \mathrm{MTZ}$, and $\mathrm{Hg}^{2+}$, respectively. 


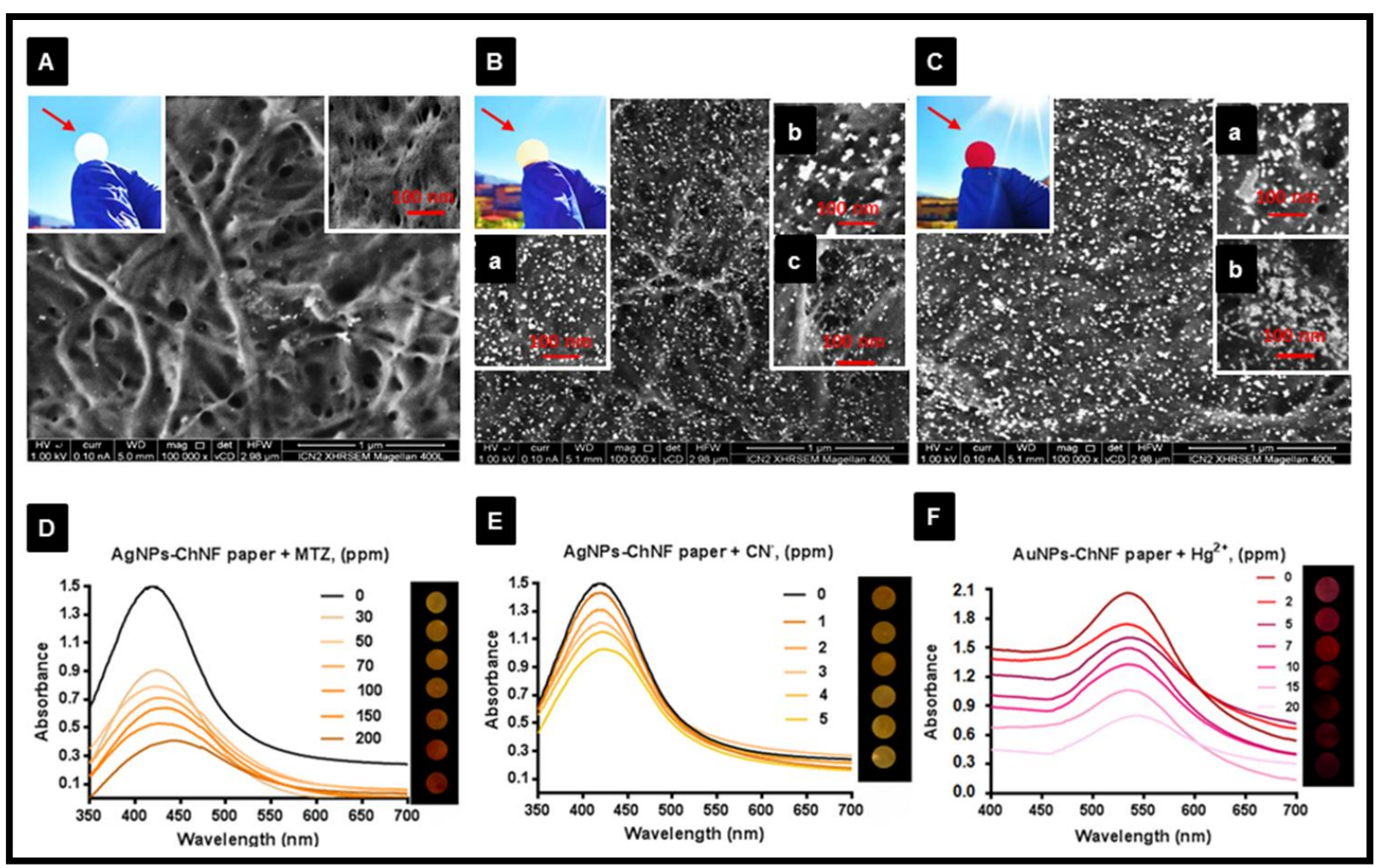

Figure 3. The fabricated plasmonic ChNF paper-based optical sensing platforms. (A) Scanning electron micrographs and a digital photograph (the inset image up-left) of bare ChNF paper. (B) Scanning electron micrographs of the fabricated AgNPs-ChNF paper: (B-a) in situ synthesized AgNPs-ChNF; (B-b) AgNPs-ChNF in the presence of $100 \mathrm{ppm}$ of MTZ; (B-C) AgNPs-ChNF paper in the presence of $3 \mathrm{ppm}$ of $\mathrm{CN}$. The inset image (up-left) is a digital photograph of the fabricated AgNPs-ChNF paper. (C) Scanning electron micrographs of the fabricated AuNPs-ChNF paper: (C-a) AuNPsChNF paper; (C-b) AuNPs-ChNF paper in the presence of $10 \mathrm{ppm}$ of $\mathrm{Hg}^{2+}$. The inset image (left) is a digital photograph of the fabricated AuNPs-ChNF paper. Changes in UV-vis spectra and corresponding colors of (D) AgNPs-ChNF paper upon addition of MTZ (0-200 ppm); (E) AgNPs-ChNF paper upon addition of $\mathrm{CN}^{-}(0-5 \mathrm{ppm})$ and (F) AuNPs-ChNF paper upon addition of $\mathrm{Hg}^{2+}$ (0-20 ppm). 


\subsection{Colorimetric ChNF paper-based optical sensing platforms}

In this work, colorimetric ChNF paper-based nanocomposites were fabricated through embedding/immobilization of curcumin (Cur) and 1, 5-diphenyl thiocarbazone (dithizone) within the ChNF paper. More details on the fabrication of colorimetric ChNF paper-based sensing platforms using curcumin and DTZ (the CurChNF paper and the DTZ-ChNF paper) are presented in the Experimental section. The yellow-orange color (Figure 4A (up)) and the UV-vis absorption spectrum (Figure 4B) of the fabricated Cur-ChNF paper with a characteristics absorption band $(\lambda \sim 440 \mathrm{~nm})$, which are related to the presence of curcumin, confirm the immobilization of curcumin into the ChNF paper. The color (Figure 4A (down)) and the UV-vis absorption spectrum (Figure 4C) of the fabricated DTZ-ChNF paper also verify the immobilization of DTZ into the ChNF paper.

Curcumin or diferuloylmethane, is a yellow-orange water-insoluble natural pigment and the active ingredient of turmeric "Curcuma longa". Curcumin is broadly used as a seasoning, coloring and protective agent in a variety of foods and drugs, as well as for treatment of various infections and diseases on account of its recognized antioxidant, antibacterial, anti-carcinogenic, anti-inflammatory and wound healing properties. Curcumin has also been employed for sensing different (bio)chemicals ${ }^{46-}$ 47. Based on studies that have been previously reported, the color and keto-enol tautomerism forms of curcumin depend on $\mathrm{pH}^{46-47}$. In the present work, as a representative example of the potential application of the fabricated Cur-ChNF paper, it was exploited as a colorimetric $\mathrm{pH}$ sensor. The color changing and UV-vis peaks of the Cur-ChNF paper at different pH values (from 7-13) are shown in Figure 4B. It is revealed from Figure 4B that the absorption intensities of the Cur-ChNF papers firstly decrease at $\lambda \sim 440 \mathrm{~nm}$ in the $\mathrm{pH}$ range of $7-10$ along with a color 
change from yellow toward red, and new red-shifted UV-vis peaks at $\lambda \sim 480 \mathrm{~nm}$ appear in the $\mathrm{pH}$ range of 11-13 with a color change from red toward brown.

DTZ is an organic chelating chemical agent that can form colored water-insoluble complexes with some metal cations ${ }^{48}$. We assessed the potential application of the fabricated DTZ-ChNF paper as a colorimetric sensor for determination of seven heavy metal ions, including $\mathrm{Zn}^{2+}, \mathrm{Co}^{2+}, \mathrm{Fe}^{3+}, \mathrm{Ni}^{2+}, \mathrm{Cd}^{2+}, \mathrm{Hg}^{2+}$, and $\mathrm{CrO}_{4}^{2-}$ based on DTZ chelation.

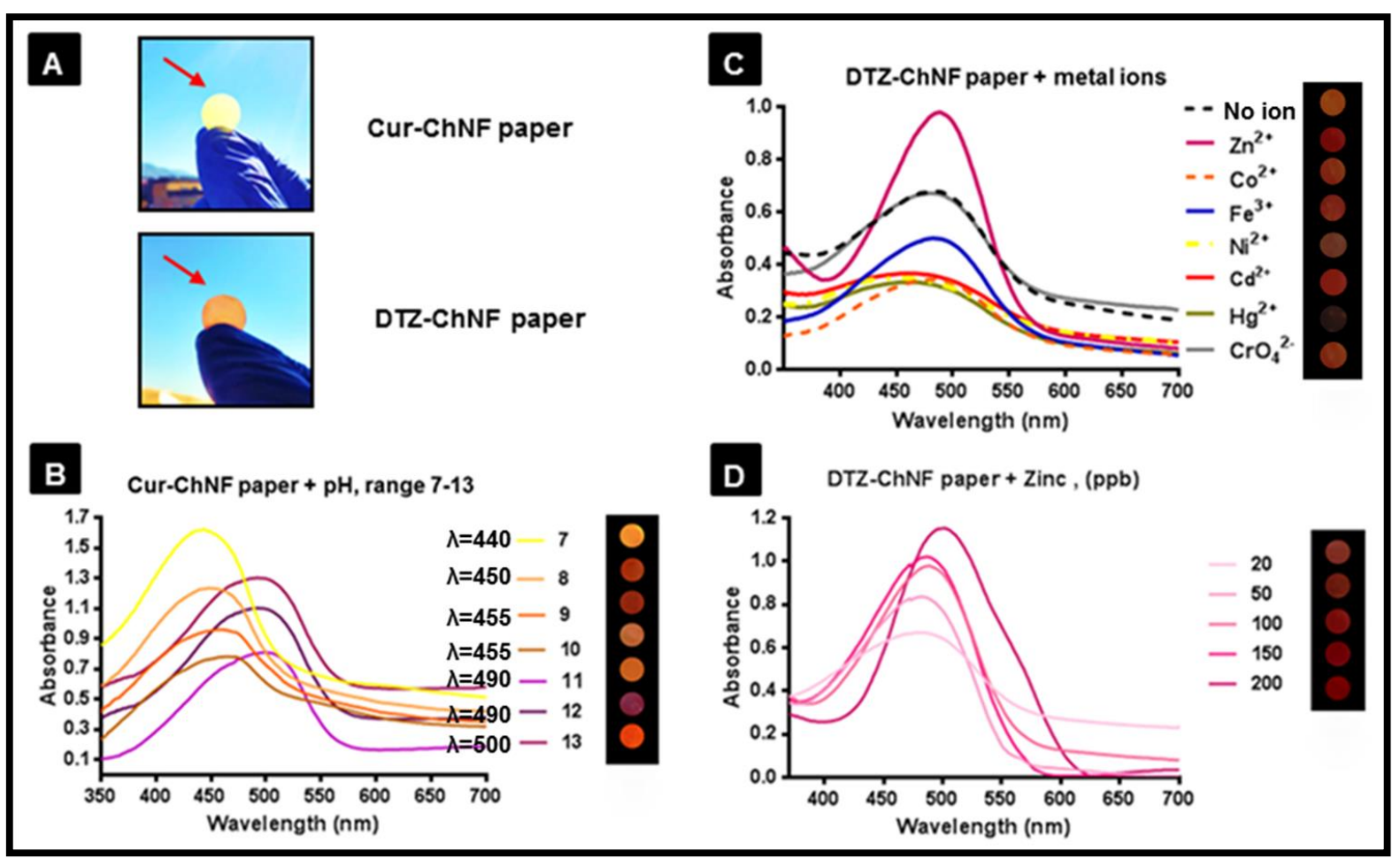

Figure 4. The fabricated colorimetric ChNF-based optical sensing bioplatforms. (A) The digital photographs of colorimetric ChNF-based nanocomposites: (up) curcuminChNF paper (Cur-ChNF paper) and (down) dithizone-ChNF paper (DTZ-ChNF paper). Changes in UV-vis spectra and corresponding colors of (B) Cur-ChNF paper at various pHs in the range of 7-13; (C) DTZ-ChNF paper upon the addition of different metal ions (100 ppb for $\mathrm{Zn}^{2+}, 1 \mathrm{ppm}$ for other metal ions), and (D) DTZ-ChNF paper upon addition of $\mathrm{Zn}^{2+}(20-200 \mathrm{ppb})$. 
Figure 4C illustrates the variations in the UV-vis absorption spectra along with corresponding colors of the fabricated DTZ-ChNF paper as a result of the addition of different metal ions (100 ppb for $\mathrm{Zn}^{2+}, 1 \mathrm{ppm}$ for other metal ions). In this case, the reaction between transition metal ions with DTZ ligand in the fabricated DTZ-ChNF paper results in formation of highly colored-complexes owing to cation-m interactions, electronic transitions, and also formation of coordination bonds between thiol group of DTZ and metal cations ${ }^{48}$. Figure 4D also shows the changes in color and the UV-vis absorption spectra of the DTZ-ChNF paper upon addition of different doses of $\mathrm{Zn}^{2+}$ ions (20-200 ppm).

\subsection{Photoluminescent ChNF paper-based optical sensing platforms}

Among different nanomaterials, photoluminescent nanoparticles (PLNPs) particularly colloidal semiconductor nanocrystals or quantum dots (QDs), carbon dots (CDs) and more recently upconversion nanoparticles (UCNPs) have given rise to enormous interest in various (bio)sensing applications on account of their unique, fascinating and tunable physicochemical features ${ }^{45,49-51}$.

QDs recognize as zero-dimensional (0-D) semiconductor nanocrystals with sizes in the range of 1-10 nm. The outstanding photophysical features of QDs such as high emission quantum yields, stable and size-tunable photoluminescence emissions, broad absorption spectra and long term photostability, which originate from the quantum confinement effect, as well as wide chemical-modification capabilities, make them among the most interesting PLNPs with significant potential in (bio)sensing applications ${ }^{50,52-54}$.

Recently, CDs as a new class of 0-D carbon nanomaterials have been widely investigated and applied in different (bio)sensing applications because of their low 
cost, low toxicity, biocompatibility, good aqueous solubility, easy fabrication, and functionalization, as well as other common photophysical properties with conventional semiconductor QDs ${ }^{49,55 .}$

Unlike QDs and CDs, which are commonly excited through UV-vis light to produce Stokes-shifted visible photoluminescence emissions, more recently a novel class of PLNPs known as upconversion nanoparticles (UCNPs) have emerged that can be excited in the near infrared (NIR) region to trigger anti-Stokes-shifted photoluminescence emission in the visible region ${ }^{51,56-58}$.

Because of unique and extraordinary optical and chemical features of UCNPS including high quantum yield, high resistance to photo-bleaching, low toxicity, longterm lifetime, narrow emission bandwidths, as well as very low optical background noise, they can be potentially considered as appealing alternatives to traditional fluorophores in design and fabrication of novel (bio)chemical sensors ${ }^{51,56-58}$.

Along with the beneficial properties of PLNPs, a pivotal step that can significantly boost their potential applications in sensors technology is the immobilization/embedding of PLNPs in appropriate sensing substrates/platforms.

In the current work, various photoluminescent NPs-ChNF paper-based nanocomposites were fabricated through embedding/immobilization of different PLNPs (including N-Acetyl L-Cysteine (NAC)-capped cadmium telluride (CdTe) quantum dots (QDs) with different emission: green-, yellow, and red-emitting QDs (GQDs, YQDs, and RQDs); thioglycolic acid (TGA) CdTe QDs with greenish-yellow emission (GyQDs); carbon dots (CDs) and amine-functionalized $\mathrm{NaYF}_{4}: \mathrm{Yb}^{3+} @ \mathrm{Er}^{3+} \& \mathrm{SiO}_{2}$ upconversion nanoparticles (UCNPs)) within the ChNF paper. Interestingly, to use ChNF paper as a biotemplate to embed the PLNPs, there is no need to modify the ChNF papers via chemical reactions owing to the presence 
of the amide and hydroxyl functional groups on the surface of ChNF paper, which can facilitate the direct coupling of amine/carboxyl-functionalized PLNPs with the ChNF paper. The fabricated QDs-ChNF based sensing platforms and the corresponding photoluminescence emissions are shown in Figure 5A. The fabricated CDs-ChNF based sensing platform and the corresponding photoluminescence emission are shown in Figure 5B. The fabricated UCNPs-ChNF-based sensing platform is also shown in Figure 5F. More details on the fabrication of different types of PL-ChNF paper-based sensing platforms are presented in the Experimental section.

As representative examples of the potential of the fabricated PLNPs-ChNF paper platforms for photoluminescent sensing applications, we assessed the CDs-ChNF paper for sensing of quercetin and the YQDs-ChNF paper for sensing of sulfide ions $\left(\mathrm{S}^{2-}\right)$. Figures $5 \mathrm{~B}$ and $5 \mathrm{C}$ illustrate the changes in photoluminescence intensity of the CDs-ChNF and YQDs-ChNF papers upon addition of different doses of quercetin (from 40 to $300 \mathrm{ppm}$ ) and sulfide ions (from 20 to $200 \mathrm{ppm}$ ), respectively. The calibration curves and LOD values corresponding to these experiments are presented in Supporting Information Figure S12. Photoluminescence spectra from the CDs-ChNF paper and YQDs-ChNF paper spots were obtained using a spectrofluoremeter (excitation wavelength $\left(\lambda_{E x}\right), 370 \mathrm{~nm}$; emission wavelengths $\left(\lambda_{E m}\right)$ for the CDs-ChNF and YQDs-ChNF papers were $445 \mathrm{~nm}$ and $560 \mathrm{~nm}$, respectively). The photoluminescence of all the fabricated PLNPs-ChNF paper nanocomposites was also modulated by graphene oxide (GO) as a well-known, highly efficient photoluminescence quenchers. 
Figures 5D-5F show vividly how the photoluminescence intensity of the fabricated CDs/YQDs/UCNPs ChNF paper-based sensing platforms is decreased upon addition of various concentrations of $\mathrm{GO}$.

Figures 5G-5L also demonstrate the confocal microscopy images of the ChNF paper and the fabricated PLNPs-ChNF papers before and after addition of GO (320 ppm), which clearly show the photoluminescence quenching caused by GO due to fluorescence resonance energy transfer (FRET) mechanism, where GO and PLNPSChNF papers operate as FRET acceptor and donor, respectively.

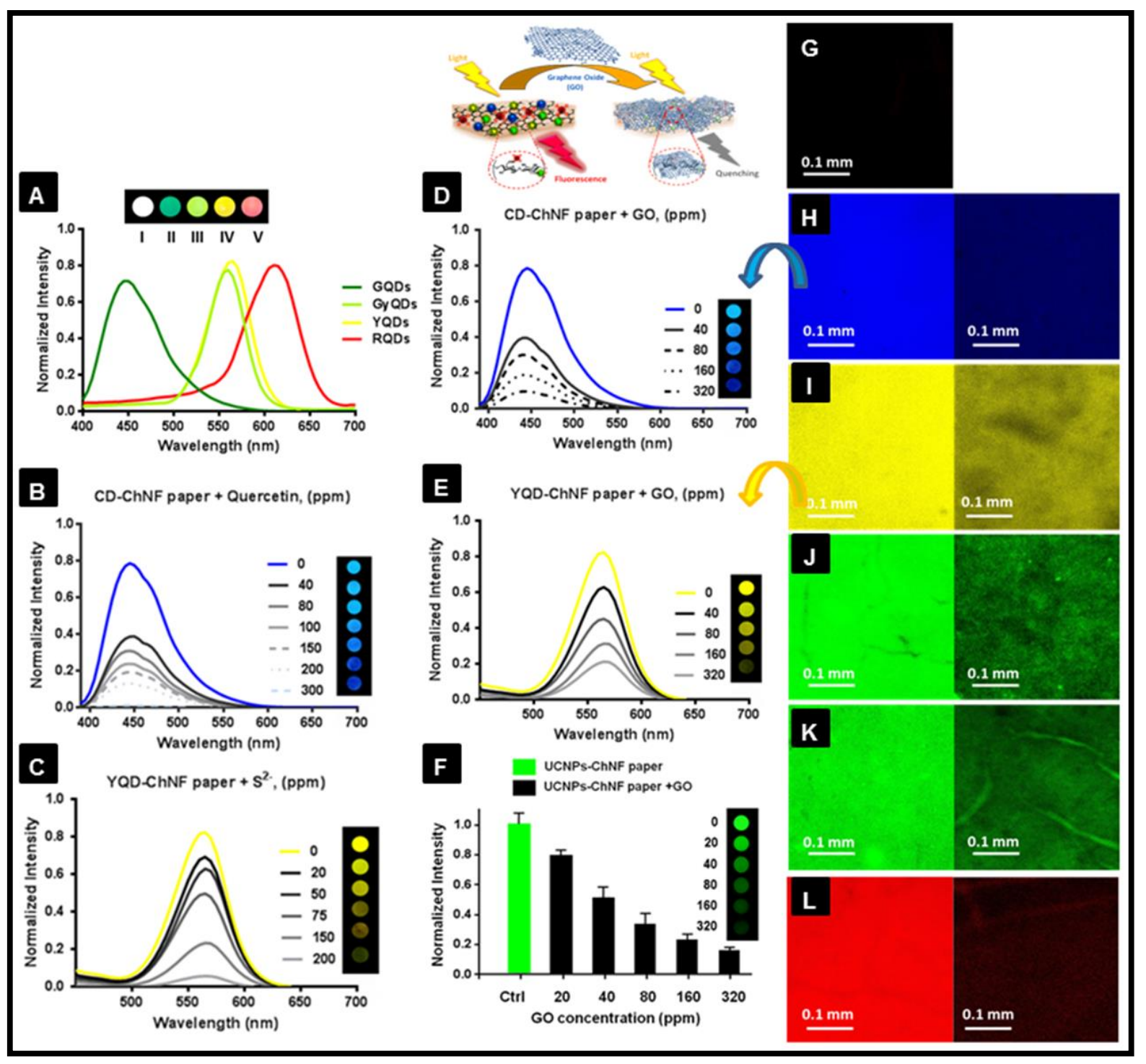


Figure 5. The photoluminescent nanoparticles-ChNF paper-based optical sensing platforms. The photoluminescence spectra and corresponding colors of the fabricated (A) N-Acetyl L-Cysteine (NAC)-functionalized cadmium telluride (CdTe) quantum dots (QDs)-ChNF paper based sensing platforms with different emission: green-emitting QDs (GQDs), yellow-emitting QDs (YQDs), red-emitting QDs (RQDs); thio-glycolic acid (TGA) CdTe QDs with greenish-yellow emission (GyQDs); (B) carbon dots (CDs)-ChNF paper upon addition of different concentrations of quercetin; (C) YQDs-ChNF paper upon addition of different concentrations of $\mathbf{S}^{2-}$. Modulation of the photoluminescence intensity of the fabricated (D) CD-ChNF paper; (E) YQD-ChNF paper and (F) amine-functionalized $\mathrm{NaYF}_{4}: \mathrm{Yb}^{3+} @ \mathrm{Er}^{3+} \& \mathrm{SiO}_{2}$ upconversion nanoparticles (UCNPs)-ChNF paper based sensing platforms by addition of graphene oxide (GO) at different concentrations. Confocal microscopy images of the fabricated (G) ChNF paper; (H) CD-ChNF paper $\left(\lambda_{E x}=370 \mathrm{~nm}\right.$, maximum $\left.\lambda_{E m} \sim 445 \mathrm{~nm}\right)$; (I) YQDChNF paper $\left(\lambda_{E x}=370 \mathrm{~nm}\right.$, maximum $\left.\lambda_{\mathrm{Em}} \sim 560 \mathrm{~nm}\right) ;(J)$ GyQD-ChNF paper $\left(\lambda_{\mathrm{Ex}}=370\right.$ $\mathrm{nm}$, maximum $\left.\lambda_{\mathrm{Em}} \sim 555 \mathrm{~nm}\right)$; $(\mathrm{K}) \mathrm{GQD}-\mathrm{ChNF}$ paper $\left(\lambda_{\mathrm{Ex}}=370 \mathrm{~nm}\right.$, maximum $\lambda_{\mathrm{Em}} \sim 450$ $\mathrm{nm})$; and (L) RQD-ChNF paper $\left(\lambda_{E x}=370 \mathrm{~nm}\right.$, maximum $\left.\lambda_{E m} \sim 610 \mathrm{~nm}\right)$ based sensing platforms before (left) and after (right) addition of GO.

\subsection{ChNF paper-based assay kits}

To demonstrate the general applicability of the ChNF paper in the fabrication of (bio)chemical assay kits, as a representative example of the potential of the ChNF paper-based sensing platforms for a wide range of sensing applications, it was exploited in the fabrication of a colorimetric assay kit for simultaneous determination of glucose, total bilirubin and albumin in human blood samples. On the other hand, given the weak microfluidic property of the ChNF paper as well as to separate plasma from whole blood samples without centrifugation, which is an essential step 
in the development of plasma-based assays, the ChNF paper was combined with plasma separation membrane and filter paper in a specially designed pattern. Full details on design and fabrication of the ChNF paper-based assay kit and its application in blood samples analysis are provided in the Experimental section and Figures $6 \mathrm{~A}$ and $6 \mathrm{~B}$. The images and calibration curves corresponding to these experiments for simultaneous colorimetric determination of glucose, total bilirubin and albumin in human blood samples using the developed smartphone-based ChNF paper assay kit are presented in Figure 6C-6E, respectively. The clinical efficiency of the developed smartphone-based ChNF paper assay kit was also validated by analyzing the blood samples of 5 volunteers and comparing their results with glucose, total bilirubin, and albumin Assay Kits as clinical reference methods. The results of both methods for glucose, total bilirubin, and albumin are plotted against each other for comparison in Figure S14A-C, respectively. The obtained regression coefficients for each analyte indicate the results of the developed smartphone-based ChNF assay kit are comparable to reference methods, which are routinely exploited in clinical laboratories for glucose, total bilirubin, and albumin detection level in blood samples. The obtained results show that the developed smartphone-based ChNF paper assay kits as user-friendly, cost-effective and portable sensors can be potentially utilized for fast and easy detection of biological analytes at the point-ofcare $(\mathrm{POC})$ as well as in the clinical laboratories. 


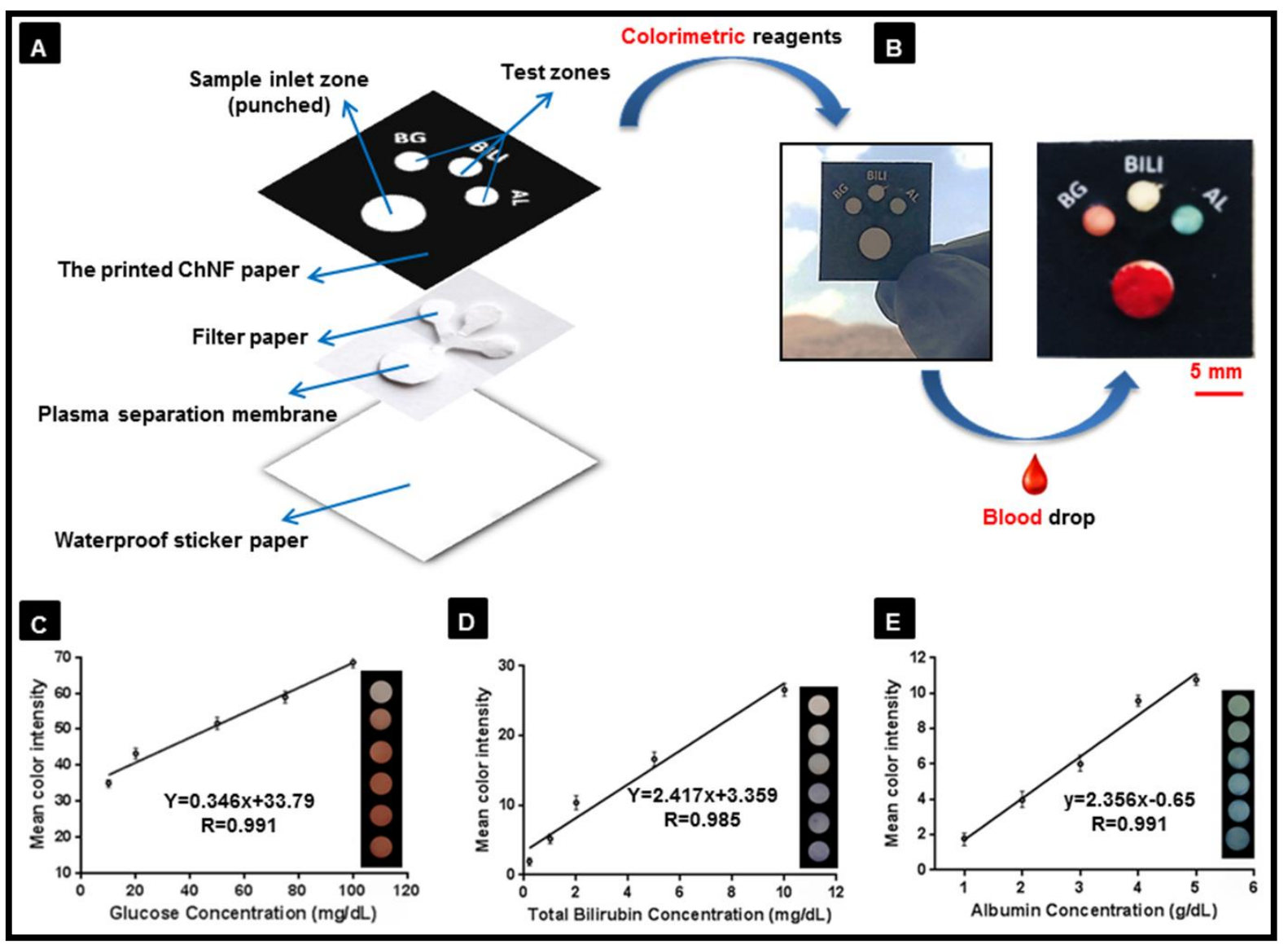

Figure 6. (A) Schematic representation for the fabrication of ChNF paper-based assay kit. (B) The digital photographs of the fabricated ChNF paper-based assay kit, which was used for colorimetric determination of glucose, total bilirubin and albumin in blood samples, before (left) and after (right) applying a drop of blood. The images and corresponding calibration curves for colorimetric determination of (C) glucose; (D) total bilirubin; and (E) albumin using the developed smartphone-based ChNF paper assay kit. (Colorimetric reagents for glucose: phosphate buffer $(\mathrm{pH}=7.5,250 \mathrm{mM})$ phenol (5 mM), 4-aminoantipyrine $(0.5 \mathrm{mM})$, glucose oxidase $>10 \mathrm{kU} /$; for total bilirubin: EDTA-Na $\mathrm{Na}_{2}(0.07 \mathrm{mM}), \quad \mathrm{NaCl}\left(6.6 \mathrm{gL}^{-1}\right)$, sulfamic acid $(70 \mathrm{mM}), 2,4-$ dichlorophenyl-diazonium salt (0.09 mM), HCl (130 mM), EDTA-Na ${ }_{2}$ (0.02 mM); for albumin: Citrate buffer $(\mathrm{pH}=4.2,30 \mathrm{mM})$, bromocresol green $(0.26 \mathrm{mM}))$. 


\subsection{Integration of loT in the developed smartphone-based ChNF paper assay kits}

Since its arrival, nanotechnology has been offered effective and valuable solutions in so many diverse fields such as biomedical, industry, agriculture and military. Moreover, inter-connection of Nano-devices and Nano-sensors with Internet has led to a new revolution in this field called "Internet of Nano Things" (IONT) ${ }^{59-60}$. Developing and establishing such technical revolution into the value chain of multifaceted areas such as healthcare requires the implementation and connection of hi-tech analytical innovations ${ }^{39}$. On the other hand, successful implementation and integration of loT technology in healthcare and especially in POC diagnostic tests include several challenges in different layers such as data storage, data management, exchange of data between devices, security and privacy, and unified and ubiquitous access. The most possible solution to aforementioned challenges is cloud computing technology and specially fog-assisted computing. Cloud computing is backbone of loT healthcare systems. It has scalability, mobility and security benefits by providing on-demand computing resources (e.g. storage, services, networks, servers, applications, and hardware) to users ${ }^{61}$.

Herein, we have designed our model of IoNT/loMT to improve potential applicability and efficiency of our developed smartphone-based assay kits via loT concept. To have a fully-integrated smartphone-based ChNF paper assay kits, we need to design different but inter-related layers based on the concept of loT healthcare systems. As a result, we added network and application layers to our model based on the presupposition of data security for all layers (Figure 7). Besides, to optimize results from our self-developed mobile's app, a machine learning layer has also been designed. 
Machine learning is closely connected to computational statistics which concentrates on making predictions using computers ${ }^{62}$. It has been witnessed huge progression in mathematical optimization and computing hardware since its coinage in 1959 by Artur Samuel ${ }^{63}$. In our proposed architecture, we have used such notion through a machine learning algorithm in Computational Analysis \& Machine Learning layer.

As illustrated in Figure 7, our loNT - IoMT based model has four but inter-related layers with some intermediating layers in and out of each layer constructed on the pre-supposition of storage (MD5: Message-digest algorithm \& RSA: Rivest-ShamirAdleman one of the first public-key cryptosystems) and communication (WPA: Wi-Fi Protected Access, WAP: Wireless Application Protocol, TLS: Transport Layer Security) security using different encryption and safety methods for different type of data usage. It also takes advantage of our self-developed application (Android \& iOS) for data processing and results obtained from fabricated ChNF paper-based sensing bioplatforms which coupled with smartphone technology for (bio)sensing applications.

The first layer is Device \& Perception layer using an access layer (2G/3G/4G/LTE/LTE-A/Broadband/Wi-Fi/WiBro/GPRS) for connectivity. Real-time processing, data visualization, embedded systems, gateways, micro-data storage, access layer are some of first layer characteristics. Besides, both storage and communication encryption and safety methods have been applied for security in this layer.

Data mined from smartphone will be transferred into Computational Analysis \& Machine Learning layer before further development in order to make predictions or decisions without being explicitly programmed to accomplish the tasks ${ }^{62}$. It would assist us to optimize our services and have better results. Decision tree-based 
machine learning algorithm has been applied to create smart data for further usages $^{63}$. Smart data created in this layer will be filtered and transcoded in Data Preprocessing layer. Computational \& big data analysis, machine learning algorithm, prediction and analysis, smart data are parts of this layer. Besides, both storage and communication encryption and safety methods have been applied for security in this layer.

Cloud, Fog and Service layer have been integrated in layer three. After preprocessing of data, it will be transferred to cloud and fog servers. Cloud servers will store patients' medical records. But their speeds are not sufficient compared to fog servers for real-time processing and giving service to end-users. Thus, fog servers will be used to solve such problems. Moreover, flow of information and data from layer one to three has designed in such a way to have a customer-oriented value chain based on their needs. Therefore, Data has been aggregated in this layer for all clinical usages. Doctors, health professionals and caregivers can take advantages of all kinds of embedded services. Besides, both storage and communication encryption and safety methods have been applied for security in this layer.

Primary focus of the present loT architecture is on medical and diagnostic tests e.g. POC diagnostic tests. Consequently, Business layer has been designed to fit into aforementioned aim. Our user-friendly self-developed mobile application is an added-value in our model and helped us to reach such a goal. Therefore, Business layer has been proposed a hybrid solution to have a better service not only for private but also for public sector as well. It has followed the same approaches in loT healthcare and loMT. As a result, we have targeted not only home-users but also experts and medical centers as our potential customers. Business ethics, Customer Relationship Management $(\mathrm{CRM})$, service management, marketing \& sales are 
important parts of this layer. Besides, both storage and communication encryption and safety methods have been applied for security in this layer.

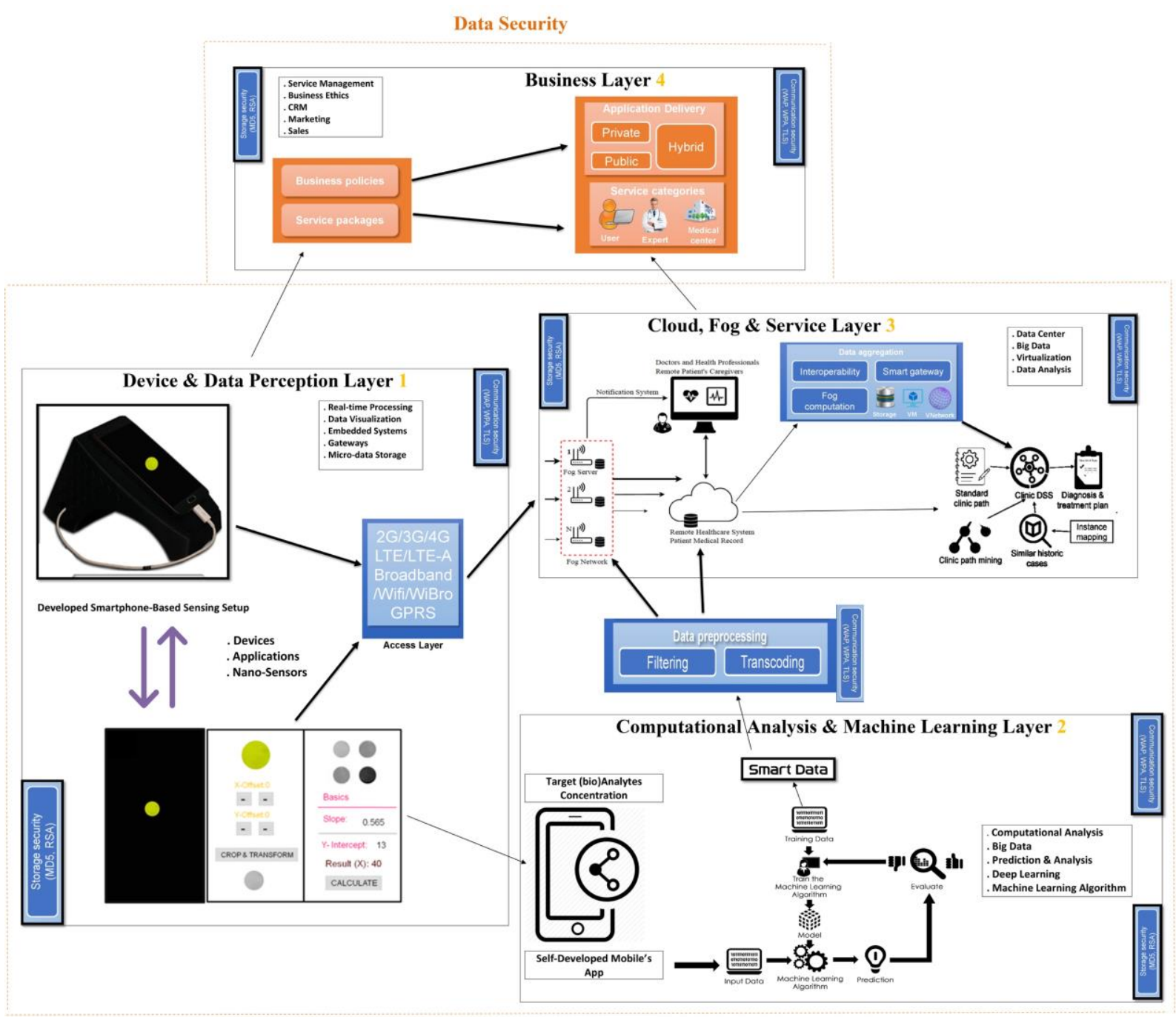

Figure 7. The proposed loT architecture the developed smartphone-based ChNF paper assay kits.

\section{CONCLUSIONS}

The present work deals with the fabrication of efficient, transparent, flexible, and biocompatible ChNF paper-based bionanocomposites. Various optical (plasmonic, photoluminescent and colorimetric) sensing bioplatforms were fabricated by taking 
the beneficial properties of ChNF paper including low cost, commercial availability, biocompatibility, biodegradability, flexibility, printability, optical transparency and other extraordinary mechanical and physicochemical properties, in combination with the unique and tunable photophysical and physicochemical properties of plasmonic NPs (AgNPs and AuNPs), PLNPs (different color emitting QDs, CDs, and UCNPs) and colorimetric reagents. A fast and simple patterning method by using an office laser printer, through printing the hydrophobic ink on the ChNF paper, was used to fabricate the ChNF paper-based optical (bio)sensing platforms with different desired patterns including 2D multi-wall, 2D cuvette patterns with hydrophilic test zones and hydrophobic walls/barriers. The applicability and efficiency of the developed ChNF paper-based platforms for analytical tasks were confirmed by optical sensing a wide range of (bio)chemicals as model analytes. Owing to the optical transparency of the developed ChNF paper-based (bio)sensors, their color/fluorescence changes upon the addition of tested analytes can be monitored visually by the naked eye and also spectroscopically using a spectrophotometer/spectrofluorometer. To digitize the images of the obtained colorimetric/fluorometric measurements using the developed ChNF paper-based sensing bioplatforms, they were coupled with mobile phones. Indeed, the coupling of ChNF paper-based sensors to smartphone technology and the designed loNT model can enhance the potential applications of these novel (bio)sensing platforms as affordable, user-friendly, and portable monitoring/sensing devices, especially in the developing smart cities.

On the other hand, the reasons why ChNF-paper can be considered as a novel, attractive and high potential substrate alongside paper and BC nanopaper for making optical sensing platforms have been presented as a comparative table (Table 1). As seen in this table, many properties of ChNF paper, from technical, 
economic and environmental points of view, are comparable and in some cases better compared to paper and BC nanopaper for making optical sensing platforms.

Building upon the unrivalled features of ChNFs as one of very promising bionanomaterial, we believe that the ChNF paper-based sensing bioplatforms have the promising potential to be exploited in the development of new strategies to fabricate simple, cost-effective, easy-to-use, green, transparent, flexible, miniaturized, and smart (bio)sensing/monitoring devices.

Table 1. Comparison of normal cellulose paper, BC nanopaper and ChNF paper properties for making optical sensing platforms from technical, economic and environmental points of view.

\begin{tabular}{|c|c|c|c|}
\hline & Normal Cellulose Paper & BC nanopaper & ChNF paper \\
\hline Production cost & Very low & Medium & Low \\
\hline $\begin{array}{l}\text { Simplicity of production } \\
\text { method }\end{array}$ & Very simple & Medium & Simple \\
\hline Raw material availability & Very high & Low & High \\
\hline Production time (For $1 \mathrm{~m}^{2}$ ) & $\begin{array}{l}\text { Very short } \\
\text { (Commercially available) }\end{array}$ & $\begin{array}{l}\text { Very long ( two weeks) } \\
\text { (Commercially available) }\end{array}$ & $\begin{array}{l}\text { Short (less than } 2 \mathrm{hr} \text { ) } \\
\text { (Commercially } \\
\text { available) }\end{array}$ \\
\hline Transparency & Non-transparent & $\sim 40 \%$ & $\sim 50 \%$ \\
\hline $\begin{array}{l}\text { Functionalizing for sensing } \\
\text { applications }\end{array}$ & Usually needed & Usually needed & Usually not needed \\
\hline Stability in aqueous media & Not stable & Stable & Stable \\
\hline $\begin{array}{l}\text { Fabrication of sensing } \\
\text { platforms/Creation of test } \\
\text { zones }\end{array}$ & $\begin{array}{l}\text { Require expensive } \\
\text { equipment and multistep } \\
\text { processing }\end{array}$ & $\begin{array}{l}\text { Very cheap, easy (one } \\
\text { step) and fast using } \\
\text { normal printers }\end{array}$ & $\begin{array}{l}\text { Very cheap, easy (one } \\
\text { step) and fast using } \\
\text { normal printers }\end{array}$ \\
\hline Optical detection methods & $\begin{array}{l}\text { Reflectance-based } \\
\text { measurements } \\
\text { (scanometric) }\end{array}$ & $\begin{array}{l}\text { Scanometric, } \\
\text { spectrophotometric, and } \\
\text { spectrofluorometric }\end{array}$ & $\begin{array}{l}\text { Scanometric, } \\
\text { spectrophotometric, and } \\
\text { spectrofluorometric }\end{array}$ \\
\hline $\begin{array}{l}\text { Compatibility with bio)chemical } \\
\text { for sensing applications }\end{array}$ & High & High & High \\
\hline Mechanical properties & Very low & Very high & Very high \\
\hline
\end{tabular}




\section{Experimental Section}

\subsection{Reagents and Equipment.}

Milli-Q ultrapure water was used in all experiments. Curcumin (CAS Number: 45837-7, $\geq 94 \%$ ), dithizone (CAS Number: 60-10-6, $\geq 98 \%$ ), methimazole (CAS Number: 60-56-0, $\geq 99 \%$ ), ethylenediamine (CAS Number: 107-15-3, $\geq 99.5 \%$ ), ethanol (CAS Number: 64-17-5, $\geq 99.8 \%$ ), tellurium powder (CAS Number: 13494-80-9, 99.997\%),

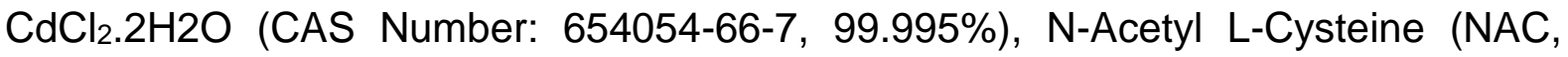
CAS Number: 616-91-1, $\geq 99 \%$ ), thioglycolic acid (TGA, CAS Number: 68-11-1, $\geq 99 \%$ ) and quercetin (CAS Number: $117-39-5$, $\geq 95 \%$ ) were obtained from Sigma Aldrich (Taufkirchen, Germany).

KCN (CAS Number: 151-50-8, $\geq 96 \%$ ), NaOH (CAS Number: 1310-73-2, $\geq 97 \%$ ), $\mathrm{AgNO}_{3}$ (CAS Number: 7761-88-8, 99.0-100.5\%), $\mathrm{HAuCl}_{4} \cdot 3 \mathrm{H}_{2} \mathrm{O}$ (CAS Number: 16961-25-4, 99-100\%), acetone (CAS Number: 67-64-1, $\geq 99.8 \%$ ), $\mathrm{FeCl}_{3} .6 \mathrm{H}_{2} \mathrm{O}$ (CAS Number: 10025-77-1, 97\%), $\mathrm{K}_{2} \mathrm{CrO}_{4}$ (CAS Number: 7789-00-6, $\geq 99.5 \%$ ), $\mathrm{ZnCl}_{2}$ (CAS Number: 7646-85-7, 98.0-100.5\%), $\mathrm{Na}_{2} \mathrm{~S} .9 \mathrm{H}_{2} \mathrm{O}$ (CAS Number: 1313-84-4, $\geq 98.0 \%$ ), $\mathrm{Ni}\left(\mathrm{NO}_{3}\right)_{2} .6 \mathrm{H}_{2} \mathrm{O}$ (CAS Number: $\left.13478-00-7, \geq 99.0 \%\right), \mathrm{Co}\left(\mathrm{NO}_{3}\right)_{2} .6 \mathrm{H}_{2} \mathrm{O}(\mathrm{CAS}$ Number: 10026-22-9, $\geq 98 \%), \quad \mathrm{Hg}\left(\mathrm{NO}_{3}\right)_{2} . \mathrm{H}_{2} \mathrm{O} \quad$ (CAS Number: 7783-34-8, $\geq 99.99 \%$ ), 3CdSO $4.8 \mathrm{H}_{2} \mathrm{O}$ (CAS Number: 7790-84-3, $\geq 98 \%$ ), and $\mathrm{NaBH}_{4}(\mathrm{CAS}$ Number: 16940$66-2, \geq 96.0 \%$ ) were purchased from Merck (Germany). Britton-Robinson buffers (pH range of $7-12$ ) were prepared through mixing phosphoric acid (Merck, Germany, CAS Number: 7664-38-2, 99\%), citric acid (Merck, Germany, CAS Number: 77-92-9, 99.5-100.5\%), and boric acid (Merck, Germany, CAS Number: 10043-35-3, 99.5100.5\%) (0.04 M each). KCI (Merck, Germany, CAS Number: 7447-40-7, $\geq 99.5 \%$ ) $(0.2 \mathrm{M})$ was used for preparation of buffer solutions in the $\mathrm{pH}$ range of $12-13$. Hydrazine hydrate (CAS Number: 7803-57-8, 100\%) was purchased from Royalex 
(India). Graphene oxide suspension was acquired from Angstron Materials, Inc. (Dayton, $\mathrm{OH}$ ).

Water-soluble N-Acetyl L-Cysteine (NAC)-capped CdTe QDs with different emission capability and also highly efficient photoluminescent CDs were produced according to the process described in the Experimental section. Chitin nanofiber paper (ChNFpaper) was supplied from Nano Novin Polymer Co., Iran. Albumin, total bilirubin, and glucose Assay Kits were supplied from Man Co., Iran. MF1, blood separation membrane, was purchased from GE Healthcare; the United States, to separate plasma from blood samples without centrifugation.

A spectrophotometer model DR5000 ${ }^{\mathrm{TM}}$ (Hach, Canada) was used to obtain the UVvis absorption spectra and absorbance measurements through placing the fabricated ChNF paper-based sensing platforms in a hand-made paperboard sample holder. The photoluminescence spectra and measurements were obtained with a Jasco Model FP-6500 Spectrofluorometer. The images were taken by Samsung Galaxy A7 (16 megapixels). The FE-SEM images and EDX analysis were obtained through a Magellan 400L High-Resolution SEM and a Quanta 650F Environmental SEM(FEI, Hillsboro, OR), respectively. High-resolution transmission electron microscopy (HRTEM) images and dynamic light scattering (DLS) analysis of synthesized CDs were performed by a transmission electron microscope (FEI, TEC9G20, USA) and a particle size analyzer (Scatteroscope I; Qudix, Inc., South Korea), respectively. Confocal microscopy was performed through a Leica TCS SPE. Thermogravimetric analysis (TGA) was performed using the thermogravimetric analyzers (Netzsch-TGA $209 \mathrm{~F} 1$, Germany) at a scan rate of $10{ }^{\circ} \mathrm{C} /$ min under $\mathrm{N}_{2}$ atmosphere. A HP LaserJet P1102 official printer was utilized to print the hydrophobic patterns on the dried ChNF papers. 


\subsection{Production of ChNF paper}

Figure $\mathrm{S} 1$ shows the experimental steps to produce ChNF paper. In brief, the shells of shrimp (Littopenaeus vannamel) was first treated with $\mathrm{HCl}$ to remove minerals and then refluxed with $\mathrm{NaOH}$ two times, as shown in Figure $\mathrm{S} 1$ to remove proteins. In the second step of alkaline treatment, 0.1 wt $\% \mathrm{H}_{2} \mathrm{O}_{2}$ was used to remove pigments. After completion of these treatments, the white shells were ground using a chicken grinder to obtain chitin powder. For mechanical nanofibrillation, the powder was converted to chitin suspension $1 \mathrm{wt} \%$ followed by passing through a super disk grinder (MKCA6-3; Masuko Sangyo Co., Ltd.) available at Nano Novin Polymer Co. (Iran). After grinding, chitin suspension was converted to the highly viscous wet gel of ChNF. The well-dispersed ChNF suspension ( $0.2 \mathrm{wt} \%$ ) was filtered by vacuuming, followed by hot pressing at $100{ }^{\circ} \mathrm{C}$ and $2 \mathrm{MPa}$ for $1 \mathrm{~h}$. This process resulted in transparent ChNF paper with a grammage of $60 \pm 3 \mathrm{~g} / \mathrm{m}^{2}$ and a density of $1.1 \mathrm{~g} / \mathrm{cm}^{3}$. The thickness of the produced ChNF paper was $\sim 45 \mu \mathrm{m}$ as shown in Figure S5A. The characterization of the produced ChNF paper is presented in the Supporting Information.

\subsection{Fabrication of plasmonic ChNF paper}

\subsubsection{Fabrication of AgNPs-ChNF paper}

The method for in situ synthesis of AgNPs within ChNF papers was represented as follows: Five pieces of dried ChNF papers $\left(6 \mathrm{~cm}^{2}\right)$ were soaked in $50 \mathrm{~mL}$ water. The $\mathrm{pH}$ of this mixture was then adjusted to 12 by addition of $\mathrm{NaOH}$ solution (2 M). After that, the reaction mixture was heated at $60{ }^{\circ} \mathrm{C}$ for $15 \mathrm{~min}$, followed by stirring. Following that, $5 \mathrm{~mL}$ of $0.1 \%(\mathrm{w} / \mathrm{v}) \mathrm{AgNO}_{3}$ solution was added dropwise (about 1 $\mathrm{mL} / \mathrm{min}$ ) into the reaction mixture under the same temperature conditions and 
continuous stirring. Then, the contents were kept under stirring and heating at $60{ }^{\circ} \mathrm{C}$ for a further $45 \mathrm{~min}$. The color change of ChNF papers from colorless to light yellow and then to amber during the synthesis procedure confirms the successful in situ synthesis of AgNPs through the gradual reduction of $\mathrm{Ag}^{+}$using ChNF paper. The contents were then cooled, and the fabricated AgNPs-ChNF papers were separated and washed thoroughly with water to ensure that any unreacted $\mathrm{Ag}^{+}$and free/weakly attached AgNPs have been completely removed from the ChNF papers. The fabricated AgNPs-ChNF papers were finally stored in the refrigerator before being used. The UV-vis absorption spectra, FE-SEM, EDX, and TGA analysis confirm the successful synthesis of AgNPs in the ChNF paper matrix. The fabricated AgNPsChNF papers were stable for more than nine months no change in their color or UVvis spectra.

\subsubsection{Fabrication of AuNPs-ChNF paper}

\subsubsection{ChNF paper as reducing agent for in situ synthesis of AuNPs}

The method for in-situ synthesis of AuNPs within ChNF paper was represented as follows: Five pieces of dried ChNF paper $\left(6 \mathrm{~cm}^{2}\right)$ were soaked in $100 \mathrm{~mL}$ of the boiling solution of $0.5 \mathrm{mM} \mathrm{HAuCl}_{4}$ in a covered beaker under stirring. The reaction mixture was kept under boiling and stirring for $2 \mathrm{~h}$. During this procedure, the adsorbed $\mathrm{AuCl}_{4}^{-}$ions in the nanonetwork scaffold of ChNF papers are gently reduced to AuNPs using the hydroxyl and amide functional groups of ChNFs, which act as reducing agents for in-situ synthesis of AuNPs within ChNF papers. The color change of ChNF papers from colorless to pale purple endorses the in situ synthesis of AuNPs within ChNF paper. The fabricated AuNPs-ChNF papers were then separated from the mixture and rinsed thoroughly with water to ensure that any 
unreacted gold ions or free/loosely attached AuNPs have been completely removed from the ChNF papers. The fabricated AuNPs-ChNF papers were finally stored in the refrigerator before being used. The UV-vis absorption spectra, FE-SEM, EDX, and TGA analysis confirm the in situ synthesis of AuNPs in the ChNF paper matrix.

\subsubsection{ChNF paper as nanonetwork embedding AuNPs}

Here, AuNPs are synthesized using hydrazine as reducing agent and then the synthesized AuNPs are embedded within the nanonetwork scaffold of ChNF paper. In brief, ten pieces of the ChNF paper spots (circle shape with a diameter of $5 \mathrm{~mm}$ ), which have been cut before with a hole punch, were immersed in $2 \mathrm{~mL}$ of $0.5 \mathrm{mM}$ $\mathrm{HAuCl}_{4}$ solution for $30 \mathrm{~min}$. After separation and rinsing the ChNF paper spots from the reaction mixture, they were soaked in $25 \mathrm{~mL}$ water followed by stirring. $500 \mu \mathrm{L}$ of $500 \mu \mathrm{M}$ hydrazine solution was then rapidly added into the reaction mixture. After 10 sec, the fabricated AuNPs-ChNF papers were rapidly separated and washed thoroughly with water to remove any free/loosely attached AuNPs. During this procedure, the color of ChNF papers changes from colorless to wine red, which confirms the presence of the synthesized AuNPs in the nanonetwork scaffold of ChNF papers. The UV-vis absorption spectra, FE-SEM, EDX, and TGA analysis also verify the fabrication of AuNPs-ChNF papers.

\subsection{Fabrication of colorimetric ChNF paper}

\subsubsection{Fabrication of Cur-ChNF paper}

To fabricate the Cur-ChNF paper, five pieces $\left(6 \mathrm{~cm}^{2}\right)$ of dried ChNF papers were immersed in $25 \mathrm{~mL}$ of curcumin solution $(0.5 \% \mathrm{w} / \mathrm{v}$ in water/acetone $6: 4(\mathrm{~V} / \mathrm{V}))$ followed by stirring at room temperature for $20 \mathrm{~min}$. In this procedure, dissolved 
curcumin molecules immobilize within the nanonetwork scaffold of the ChNF papers, which is subsequently verified by the color change of the colorless ChNF papers to yellow-orange (color of curcumin). After that, the ChNF paper pieces containing embedded curcumin molecules (Cur-ChNF paper) were separated and washed several times with water to remove any unimmobilized curcumin from the ChNF papers. The fabricated Cur-ChNF papers were kept in the refrigerator before being used. The fabrication of Cur-ChNF paper was also verified by UV-visible absorption spectra. The fabricated Cur-ChNF papers were stable for more than nine months no change in their color or UV-vis spectra.

\subsubsection{Fabrication of DTZ-ChNF paper}

To fabricate the DTZ-ChNF paper, five pieces $\left(6 \mathrm{~cm}^{2}\right)$ of dried ChNF papers were immersed in $20 \mathrm{~mL}$ of ethanolic solution of $0.1 \% \mathrm{w} / \mathrm{v}$ dithizone (DTZ) followed by gentle stirring for $30 \mathrm{~min}$. Then, the ChNF paper pieces containing embedded DTZ (DTZ-ChNF paper) were separated and completely washed with water several times to eliminate any unimmobilized DTZ from the ChNF papers. The UV-vis peak and color of the fabricated DTZ-ChNF paper confirm the immobilization of DTZ into the ChNF paper. The fabricated DTZ-ChNF paper was stable for more than nine months no change in its color or UV-vis spectrum.

\subsection{Fabrication of photoluminescent (QDs/CDs/ UCNPs) ChNF papers}

In a typical procedure to embed CDs in the ChNF papers, two pieces $\left(6 \mathrm{~cm}^{2}\right)$ of dried ChNF papers were soaked in $5 \mathrm{~mL}$ of the 10-times diluted solution of the assynthesized CDs followed by gentle stirring for $60 \mathrm{~min}$. The fabricated CDs-ChNF 
papers were separated and then rinsed with water several times to remove any unattached/unimmobilized CDs from the CDs-ChNF papers. The fabricated CDsChNF papers were stored in the refrigerator before being used.

The procedures for fabrication of other PLNPs-ChNF papers (QDs/UCNPs-ChNF papers) through embedding/immobilization of different functionalized QDs (including N-Acetyl L-Cysteine (NAC) functionalized CdTe QDs with different emission: green-, yellow, and red-emitting QDs (GQDs, YQDs, and RQDs; Thioglycolic acid (TGA) CdTe QDs with greenish-yellow emission (GyQDs)) and amine-functionalized $\mathrm{NaYF}_{4}: \mathrm{Yb}^{3+} @ \mathrm{Er}^{3+} \& \mathrm{SiO}_{2}$ upconversion nanoparticles (UCNPs) within the ChNF papers are similar to the aforementioned procedure for fabrication of CDs-ChNF papers, except the as-synthesized PLNPs solutions are used instead of CDs solution.

\subsection{Design and fabrication of ChNF paper-based sensing platforms}

To use the fabricated ChNF paper-based nanocomposites as templates for (bio)sensing, it is necessary to create the test zones on their surface. To meet this end, various patterns with specific patterned layouts (2D multi-wall and 2D cuvette patterns) with hydrophobic walls/barriers and hydrophilic test zones/channels were created using an office laser printer via direct-printing the hydrophobic ink on the fabricated ChNF paper-based nanocomposites. Initially, the desired patterns were created in Microsoft Office Word (2015) and then printed by an office printer on the dried films of the fabricated ChNF paper-based nanocomposites, which have already been attached on normal paper by an adhesive tape. The printed ChNF paper-based sensing platforms were then separated from the paper. Figure $3 \mathrm{E}$ depicts the hydrophilic (colorless parts) and hydrophobic (black parts) areas of the printed ChNF 
paper-based sensing platforms. The percolation of the colored water drops into the hydrophilic area (yellow part) and their lack of percolation into the hydrophobic area because of the ink/toner coating, confirm the creation of hydrophilic test zones (with hydrophobic barriers) on the surface of fabricated ChNF paper-based sensing platforms. The printed ChNF paper-based sensing platforms were finally stored in dark conditions before being used. The ChNF paper-based nanocomposites dried films can also be cut by using a hole punch to fabricate spot patterns for individual assays (see Figure 3C).

\subsection{Recommended procedure for detection of (bio)chemicals utilizing the fabricated ChNF paper-based sensing platforms}

$5 \mu \mathrm{L}$ (for 2D multi-wall and spot designs) and $20 \mu \mathrm{L}$ (for 2D cuvette design) of analytes solutions were dropped to each of the test zones of the fabricated ChNF paper-based sensing platforms and then allowed to dry for $15 \mathrm{~min}$ at room temperature. For spectrophotometric or spectrofluorometric determination of analytes, the photoluminescence and UV-vis absorption spectra/intensities of the test zones were measured, after inserting them in hand-made paperboard sample holders. For visual or scanometric determination, the images, color intensities and corresponding determined concentrations of the test zones were taken and processed by using the 3D-printed smartphone-based sensing setup.

\subsection{Fabrication of 3D-printed smartphone-based sensing setup}

A dark chamber, as seen in Figure S13, with well-defined places for smartphone camera and UV/white lamps was fabricated using a 3D printer ${ }^{34}$. Two UV LED lamps with $\lambda=365 \mathrm{~nm}$ (for excitation of the fabricated QDs/CDs-ChNF papers), and 
two white LED lamps from Epileds, Taiwan with $3.3 \mathrm{~V}$, which placed in defined places of the fabricated smartphone platform, were used for PL and colorimetric sensing, respectively. The photoluminescence of the fabricated UCNPs-ChNF paper was appeared using a $980 \mathrm{~nm}$ Infrared Line Laser Module with output power 150 $\mathrm{mW}$ and power supply 3mA from SHHO, China, which placed in the defined place of the fabricated smartphone platform. To take the photoluminescence of the fabricated UCNPs-ChNF paper, the 3D-printed smartphone-based platform was equipped with a UV/IR cut filter to block the NIR light. The assembled smatphone-based setup is depicted in Figure S13. The fabricated ChNF paper-based sensing platforms were first placed within the dark chamber via a strip hole. Lamps were powered using a smartphone via a USB port. A self-developed mobile application was also used to quantitate the changes in the color intensities of the fabricated ChNF paper-based sensing platforms, which were taken by integrated smartphone's camera, related to the concentration of the tested analytes (See Figure S13).

\subsection{Fabrication and application of ChNF paper-based assay kit for blood sample analysis}

For fabrication of ChNF paper-based assay kit, as seen in Figure 6A, the blood separation membrane MF1 (circle shaped with $5 \mathrm{~mm}$ diameter) and filter paper Whatman No.1 with the designed pattern were first attached on a waterproof sticker paper. The printed ChNF paper with a specific pattern (three circle-shaped test zones (with $3 \mathrm{~mm}$ diameter) for glucose, total bilirubin and albumin; and a punched part (with $5 \mathrm{~mm}$ diameter) as sample inlet zone, which designed for the blood separation membrane) was then placed on the attached set. After fabrication of the assay kit substrate, $5 \mu \mathrm{L}$ of the pre-mixed colorimetric reagents for glucose 
(phosphate buffer $(\mathrm{pH}=7.5,250 \mathrm{mM})$, phenol $(5 \mathrm{mM})$, 4-aminoantipyrine $(0.5 \mathrm{mM})$, glucose oxidase $>10 \mathrm{kU} /$ ), total bilirubin (EDTA-Na2 $(0.07 \mathrm{mM}), \mathrm{NaCl}\left(6.6 \mathrm{gL}^{-1}\right)$, sulfamic acid (70 mM), 2,4-dichlorophenyl-diazonium salt (0.09 mM), $\mathrm{HCl}(130 \mathrm{mM})$, EDTA-Na2 $(0.02 \mathrm{mM}))$ and albumin (citrate buffer $(\mathrm{pH}=4.2,30 \mathrm{mM})$, bromocresol green $(0.26 \mathrm{mM}))$ were separately drop-dried on each corresponding test zones of the fabricated ChNF paper-based assay kit. To evaluate the clinical applicability of the developed smartphone-based ChNF paper assay kit, as seen in Figure 6B one drop of blood was applied directly to the sample inlet zone of the fabricated assay kit and then subjected for colorimetric determination of glucose, total bilirubin, and albumin.

\subsection{Synthesis of PLNPs}

\subsubsection{Synthesis of N-Acetyl L-Cysteine (NAC)-capped CdTe QDs (GQDs,} YQDs and RQDs) NAC-capped CdTe QDs with different emission colors (green, yellow and red) were synthesized according to our previously reported method after slight modifications of the method described by Liu et al. ${ }^{64-65}$. In brief, $2.87 \mathrm{mmol}$ of NAC was first added to $10 \mathrm{~mL}$ of $\mathrm{CdCl}_{2}$ solution $(0.290 \mathrm{M})$, and its $\mathrm{pH}$ was adjusted to 9 by $\mathrm{NaOH}$ solution. Following that, the reaction mixture was stirred for 5 min under argon flow. Tellurium powder $(0.76 \mathrm{mmol})$ was then added to $75 \mathrm{~mL}$ of $64 \mathrm{mM}$ $\mathrm{NaBH}_{4}$ solution at $50{ }^{\circ} \mathrm{C}$ in a three-neck round bottom flask under argon atmosphere. After $20 \mathrm{~min}, 10 \mathrm{~mL}$ of $\mathrm{CdCl}_{2}$ solution $(\mathrm{pH}=9)$ was added, followed by temperature increasing to $150{ }^{\circ} \mathrm{C}$. NAC-capped CdTe QDs with different emission colors were obtained at different reaction times, followed by cooling in an ice bath. Photoluminescence spectra of as-prepared CdTe QDs with peaks placed at 450, 560 and $610 \mathrm{~nm}$ were recorded for GQDs, YQDs, and RQDs, respectively. 


\subsubsection{Synthesis of thio - glycolic acid (TGA) capped CdTe QDs (GyQDs)}

TGA capped CdTe QDs were synthesized according to the procedure described above for the synthesis of NAC-capped CdTe QDs by using $2.87 \mathrm{mmol}$ of TGA instead of NAC ${ }^{66}$.

\subsubsection{Synthesis of CDs}

Highly PL CDs were synthesized by mixing $500 \mathrm{mg}$ citric acid and $500 \mu \mathrm{L}$ ethylenediamine in $10 \mathrm{~mL}$ water. Afterward, the reaction mixture was transferred to a $30 \mathrm{~mL}$ autoclave (Teflon-lined stainless-steel) and then heated at $200{ }^{\circ} \mathrm{C}$ for 5 hours 34, 66. The HRTEM images, DLS analysis, and photoluminescence spectrum of the synthesized CDs are depicted in Figure S15.

\subsubsection{Synthesis of upconversion NPs}

The synthesis and characterization of amine-functionalized $\mathrm{NaYF}_{4}: \mathrm{Yb}^{3+} @ \mathrm{Er}^{3+} \& \mathrm{SiO}_{2}$ upconversion NPs is provided in Supporting Information.

\section{Supporting Information}

The Supporting Information is available free of charge on the ACS Publications website at ....

- Additional details on characterization of ChNF paper (experimental steps, AFM images, XRD analysis, EDX spectra, TGA analysis, and tensile stressstrain curve), plasmonic ChNF paper (cross-section/FE-SEM and AFM images, EDX spectra, TGA analysis, estimation of the size distribution, and UV-vis spectrum of in situ synthesized AuNPs-ChNF paper), synthesized CDs (HRTEM images, photoluminescence spectrum and DLS analysis); synthesis 
and characterization of upconversion $\quad \mathrm{NaYF}_{4}: \mathrm{Yb}^{3+} / \mathrm{Er}^{3+} @ \mathrm{SiO}_{2}-\mathrm{NH}_{2}$ nanoparticles (TEM images and histograms, ATR FT-IR and Photoluminescent spectra). Also, details about analytical figures of merit and the influence of coexisting substances for determination of $\mathrm{MTZ}, \mathrm{CN}^{-}$, and $\mathrm{Hg}^{2+}$ using the plasmonic ChNF paper; calibration plots of determination of MTZ, $\mathrm{CN}^{-}, \mathrm{Hg}^{2+}$, quercetin and $\mathrm{S}^{2-}$; the illustration of the 3D-printed smartphone-based sensing setup, the results related to correlation between obtained results of glucose, total bilirubin, and albumin analysis using the fabricated ChNF paper-based assay kit and reference methods and (Table S1 and Figures S1-S18).

\section{ACKNOWLEDGMENT}

Financial support from Iran's National Elites Foundation (INEF), Chemistry \& Chemical Engineering Research Centre of Iran (Tehran, Iran), Nano Novin Polymer Co. (Iran), and the Nano Match program of Iran Nanotechnology Initiative Council (INIC) are gratefully acknowledged. The ICN2 is funded by the CERCA Programme / Generalitat de Catalunya. The ICN2 is supported by the Severo Ochoa program of the Spanish Ministry of Economy, Industry and Competitiveness (MINECO, grant No. SEV-2017-0706 and MAT2017-87202-P). The financial support of "the Czech Science Foundation (No. 19-00676S)" is also greatly appreciated.

\section{NOTES}

The authors declare no competing financial interest. 


\section{AUTHOR CONTRIBUTIONS}

T.N. and H.G. conceived of the original idea and planned the experiments. T.N.,

H.G., and A.M. analyzed the data. T.N. carried out the experiment. T.N. and H.G. wrote the manuscript. H.Y. produced and characterized the ChNF paper samples and wrote the related part of the manuscript. U.K. and D.H. synthesized and characterized the upconversion NPs and wrote the related part of the manuscript. M.H. designed the loT model and wrote the related part of the manuscript. H.G. and A.M. supervised the overall project. All authors discussed the results and contributed to the final manuscript.

\section{REFERENCES}

(1) Babu, R. P.; O'connor, K.; Seeram, R. Current Progress on Bio-based Polymers and Their Future Trends. Progress in Biomaterials 2013, 2 (1), 8.

(2) Grossman, R. F.; Nwabunma, D. Biopolymer nanocomposites: processing, properties, and applications, John Wiley \& Sons: 2013; Vol. 8.

(3) Golmohammadi, H.; Morales-Narvaez, E.; Naghdi, T.; Merkoci, A. Nanocellulose in Sensing and Biosensing. Chemistry of Materials 2017, 29 (13), 5426-5446.

(4) Aranaz, I.; Mengíbar, M.; Harris, R.; Paños, I.; Miralles, B.; Acosta, N.; Galed, G.; Heras, Á. Functional characterization of chitin and chitosan. Current chemical biology 2009, 3 (2), 203-230.

(5) Kurita, K. Chitin and chitosan: functional biopolymers from marine crustaceans. Marine Biotechnology 2006, 8 (3), 203-226.

(6) Huang, W. Chitin Nanopapers. In Nanopapers; Elsevier: 2018; pp 175-200.

(7) Jin, J.; Lee, D.; Im, H. G.; Han, Y. C.; Jeong, E. G.; Rolandi, M.; Choi, K. C.; Bae, B. S. Chitin nanofiber transparent paper for flexible green electronics. Advanced Materials 2016, 28 (26), 5169-5175.

(8) Jayakumar, R.; Prabaharan, M.; Nair, S.; Tamura, H. Novel chitin and chitosan nanofibers in biomedical applications. Biotechnology advances 2010, 28 (1), 142-150.

(9) Kim, J.-K.; Kim, D. H.; Joo, S. H.; Choi, B.; Cha, A.; Kim, K. M.; Kwon, T.-H.; Kwak, S. K.; Kang, S. J.; Jin, J. Hierarchical chitin fibers with aligned nanofibrillar architectures: A nonwoven-mat separator for lithium metal batteries. ACS nano 2017, 11 (6), 6114-6121.

(10) Fan, Y.; Fukuzumi, H.; Saito, T.; Isogai, A. Comparative characterization of aqueous dispersions and cast films of different chitin nanowhiskers/nanofibers. International journal of biological macromolecules 2012, 50 (1), 69-76.

(11) Ifuku, S. Chitin and chitosan nanofibers: Preparation and chemical modifications. Molecules 2014, 19 (11), 18367-18380.

(12) Lin, N.; Huang, J.; Dufresne, A. Preparation, properties and applications of polysaccharide nanocrystals in advanced functional nanomaterials: a review. Nanoscale 2012, 4 (11), 3274-3294.

(13) Ifuku, S.; Nomura, R.; Morimoto, M.; Saimoto, H. Preparation of chitin nanofibers from mushrooms. Materials 2011, 4 (8), 1417-1425.

(14) Ifuku, S.; Shervani, Z.; Saimoto, H. Chitin nanofibers, preparations and applications. In Advances in Nanofibers; IntechOpen: 2013. 
(15) Ifuku, S.; Saimoto, H. Chitin nanofibers: preparations, modifications, and applications. Nanoscale 2012, 4 (11), 3308-3318.

(16) Zhang, X.; Rolandi, M. Engineering strategies for chitin nanofibers. Journal of Materials Chemistry B 2017, 5 (14), 2547-2559.

(17) Jayakumar, R.; Chennazhi, K. P.; Srinivasan, S.; Nair, S. V.; Furuike, T.; Tamura, H. Chitin scaffolds in tissue engineering. International journal of molecular sciences 2011, 12 (3), 1876-1887.

(18) Jayakumar, R.; Menon, D.; Manzoor, K.; Nair, S.; Tamura, H. Biomedical applications of chitin and chitosan based nanomaterials-A short review. Carbohydrate polymers 2010, 82 (2), 227-232.

(19) Azuma, K.; Ifuku, S. Nanofibers based on chitin: a new functional food. Pure and Applied Chemistry 2016, 88 (6), 605-619.

(20) Dutta, J.; Tripathi, S.; Dutta, P. Progress in antimicrobial activities of chitin, chitosan and its oligosaccharides: a systematic study needs for food applications. Food Science and Technology International 2012, 18 (1), 3-34.

(21) Crini, G. Recent developments in polysaccharide-based materials used as adsorbents in wastewater treatment. Progress in polymer science 2005, 30 (1), 38-70.

(22) Mattioli-Belmonte, M.; Zizzi, A.; Lucarini, G.; Giantomassi, F.; Biagini, G.; Tucci, G.; Orlando, F.; Provinciali, M.; Carezzi, F.; Morganti, P. Chitin nanofibrils linked to chitosan glycolate as spray, gel, and gauze preparations for wound repair. Journal of Bioactive and Compatible Polymers 2007, 22 (5), 525-538.

(23) Muzzarelli, R. A.; Morganti, P.; Morganti, G.; Palombo, P.; Palombo, M.; Biagini, G.; Belmonte, M. M.; Giantomassi, F.; Orlandi, F.; Muzzarelli, C. Chitin nanofibrils/chitosan glycolate composites as wound medicaments. Carbohydrate Polymers 2007, 70 (3), 274-284.

(24) Tsutsumi, Y.; Koga, H.; Qi, Z.-D.; Saito, T.; Isogai, A. Nanofibrillar chitin aerogels as renewable base catalysts. Biomacromolecules 2014, 15 (11), 4314-4319.

(25) Parolo, C.; Merkoçi, A. based nanobiosensors for diagnostics. Chemical Society Reviews 2013, 42

(2), 450-457.

(26) Mahadeva, S. K.; Walus, K.; Stoeber, B. Paper as a platform for sensing applications and other devices: A review. ACS applied materials \& interfaces 2015, 7 (16), 8345-8362.

(27) Akyazi, T.; Basabe-Desmonts, L.; Benito-Lopez, F. Review on microfluidic paper-based analytical devices towards commercialisation. Analytica chimica acta 2018, 1001, 1-17.

(28) Meredith, N. A.; Quinn, C.; Cate, D. M.; Reilly, T. H.; Volckens, J.; Henry, C. S. based analytical devices for environmental analysis. Analyst 2016, 141 (6), 1874-1887.

(29) Liana, D. D.; Raguse, B.; Gooding, J. J.; Chow, E. Recent advances in paper-based sensors. sensors 2012, 12 (9), 11505-11526.

(30) Yang, Y.; Noviana, E.; Nguyen, M. P.; Geiss, B. J.; Dandy, D. S.; Henry, C. S. based microfluidic devices: emerging themes and applications. Analytical Chemistry 2016, 89 (1), 71-91.

(31) Naghdi, T.; Golmohammadi, H.; Vosough, M.; Atashi, M.; Saeedi, I.; Maghsoudi, M. T. Lab-onnanopaper: An optical sensing bioplatform based on curcumin embedded in bacterial nanocellulose as an albumin assay kit. Analytica chimica acta 2019, 1070, 104-111.

(32) Morales-Narváez, E.; Golmohammadi, H.; Naghdi, T.; Yousefi, H.; Kostiv, U.; Horak, D.; Pourreza, N.; Merkoçi, A. Nanopaper as an optical sensing platform. ACS nano 2015, 9 (7), 7296-7305.

(33) Pourreza, N.; Golmohammadi, H.; Naghdi, T.; Yousefi, H. Green in-situ synthesized silver nanoparticles embedded in bacterial cellulose nanopaper as a bionanocomposite plasmonic sensor. Biosensors and Bioelectronics 2015, 74, 353-359.

(34) Tabatabaee, R. S.; Golmohammadi, H.; Ahmadi, S. H. Easy Diagnosis of Jaundice: A Smartphonebased Nanosensor Bioplatform using Photoluminescent Bacterial Nanopaper for Point-of-Care Diagnosis of Hyperbilirubinemia. ACS sensors 2019, 4 (4), 1063-1071.

(35) Ashton, K. That 'internet of things' thing. RFID journal 2009, 22 (7), 97-114.

(36) Atzori, L.; lera, A.; Morabito, G. Understanding the Internet of Things: definition, potentials, and societal role of a fast evolving paradigm. Ad Hoc Networks 2017, 56, 122-140. 
(37) Abidi, B.; Jilbab, A.; Haziti, M. E. L. In Wireless Sensor Networks in Biomedical: Wireless Body Area Networks, Cham, Springer International Publishing: Cham, 2017; pp 321-329.

(38) Minoli, D.; Occhiogrosso, B. Blockchain mechanisms for loT security. Internet of Things 2018, 1, 1-13.

(39) Mayer, M.; Baeumner, A. J. A Megatrend Challenging Analytical Chemistry: Biosensor and Chemosensor Concepts Ready for the Internet of Things. Chemical reviews 2019, 119 (13), 79968027.

(40) Nayyar, A.; Puri, V.; Le, D.-N. Internet of Nano Things (IoNT): Next Evolutionary Step in Nanotechnology. 2017, 7, 4-8, DOI: 10.5923/j.nn.20170701.02.

(41) Akyildiz, I. F.; Jornet, J. M. The internet of nano-things. Wireless Commun. 2010, 17 (6), 58-63, DOI: 10.1109/mwc.2010.5675779.

(42) Anker, J. N.; Hall, W. P.; Lyandres, O.; Shah, N. C.; Zhao, J.; Van Duyne, R. P. Biosensing with plasmonic nanosensors. In Nanoscience and Technology: A Collection of Reviews from Nature Journals; World Scientific: 2010; pp 308-319.

(43) Homola, J. Surface plasmon resonance sensors for detection of chemical and biological species. Chemical reviews 2008, 108 (2), 462-493.

(44) Mayer, K. M.; Hafner, J. H. Localized surface plasmon resonance sensors. Chemical reviews 2011, 111 (6), 3828-3857.

(45) Bigdeli, A.; Ghasemi, F.; Golmohammadi, H.; Abbasi-Moayed, S.; Nejad, M. A. F.; Fahimi-Kashani, N.; Jafarinejad, S.; Shahrajabian, M.; Hormozi-Nezhad, M. R. Nanoparticle-based optical sensor arrays. Nanoscale 2017, 9 (43), 16546-16563.

(46) Sharma, R.; Gescher, A.; Steward, W. Curcumin: the story so far. European journal of cancer 2005, 41 (13), 1955-1968.

(47) Pourreza, N.; Golmohammadi, H. Application of curcumin nanoparticles in a lab-on-paper device as a simple and green $\mathrm{pH}$ probe. Talanta 2015, 131, 136-141.

(48) Irving, H.; Iwantscheff, G. The analytical applications of dithizone. Analytical Chemistry 1980, 8 (4), 321-366.

(49) Lim, S. Y.; Shen, W.; Gao, Z. Carbon quantum dots and their applications. Chemical Society Reviews 2015, 44 (1), 362-381.

(50) Costa-Fernández, J. M.; Pereiro, R.; Sanz-Medel, A. The use of luminescent quantum dots for optical sensing. TrAC Trends in Analytical Chemistry 2006, 25 (3), 207-218.

(51) Chen, G.; Qiu, H.; Prasad, P. N.; Chen, X. Upconversion nanoparticles: design, nanochemistry, and applications in theranostics. Chemical reviews 2014, 114 (10), 5161-5214.

(52) Raymo, F. M.; Yildiz, I. Luminescent chemosensors based on semiconductor quantum dots. Physical Chemistry Chemical Physics 2007, 9 (17), 2036-2043.

(53) Hildebrandt, N.; Spillmann, C. M.; Algar, W. R.; Pons, T.; Stewart, M. H.; Oh, E.; Susumu, K.; Diaz, S. A.; Delehanty, J. B.; Medintz, I. L. Energy transfer with semiconductor quantum dot bioconjugates: a versatile platform for biosensing, energy harvesting, and other developing applications. Chemical reviews 2016, $117(2), 536-711$.

(54) Pisanic li, T.; Zhang, Y.; Wang, T. Quantum dots in diagnostics and detection: principles and paradigms. Analyst 2014, 139 (12), 2968-2981.

(55) Wang, Y.; Hu, A. Carbon quantum dots: synthesis, properties and applications. Journal of Materials Chemistry C 2014, 2 (34), 6921-6939.

(56) Gu, B.; Zhang, Q. Recent advances on functionalized upconversion nanoparticles for detection of small molecules and ions in biosystems. Advanced Science 2018, 5 (3), 1700609.

(57) Vilela, P.; El-Sagheer, A.; Millar, T. M.; Brown, T.; Muskens, O. L.; Kanaras, A. G. Graphene oxideupconversion nanoparticle based optical sensors for targeted detection of mRNA biomarkers present in Alzheimer's disease and prostate cancer. ACS sensors 2016, 2 (1), 52-56.

(58) You, M.; Lin, M.; Gong, Y.; Wang, S.; Li, A.; Ji, L.; Zhao, H.; Ling, K.; Wen, T.; Huang, Y. Household fluorescent lateral flow strip platform for sensitive and quantitative prognosis of heart failure using dual-color upconversion nanoparticles. ACS nano 2017, 11 (6), 6261-6270. 
(59) Nayyar, A.; Puri, V.; Le, D.-N. Internet of nano things (IoNT): Next evolutionary step in nanotechnology. Nanoscience and Nanotechnology 2017, 7 (1), 4-8.

(60) Akyildiz, I. F.; Jornet, J. M. The internet of nano-things. IEEE Wireless Communications 2010, 17 (6), 58-63.

(61) Sultan, N. Making use of cloud computing for healthcare provision: Opportunities and challenges. International Journal of Information Management 2014, 34 (2), 177-184.

(62) de Mello, R. F.; Ponti, M. A. Machine Learning: A Practical Approach on the Statistical Learning Theory, Springer: 2018.

(63) Doupe, P.; Faghmous, J.; Basu, S. Machine Learning for Health Services Researchers. Value in Health 2019, 22 (7), 808-815.

(64) Liu, J.; Li, G.; Yang, X.; Wang, K.; Li, L.; Liu, W.; Shi, X.; Guo, Y. Exciton Energy Transfer-Based Quantum Dot Fluorescence Sensing Array: “Chemical Noses" for Discrimination of Different Nucleobases. Analytical chemistry 2014, 87 (2), 876-883.

(65) Abbasi-Moayed, S.; Golmohammadi, H.; Bigdeli, A.; Hormozi-Nezhad, M. R. A rainbow ratiometric fluorescent sensor array on bacterial nanocellulose for visual discrimination of biothiols. Analyst 2018, 143 (14), 3415-3424.

(66) Ghasemi, F.; Hormozi-Nezhad, M. R.; Mahmoudi, M. Identification of catecholamine neurotransmitters using fluorescence sensor array. Analytica chimica acta 2016, 917, 85-92.

\section{For Table of Contents Only:}

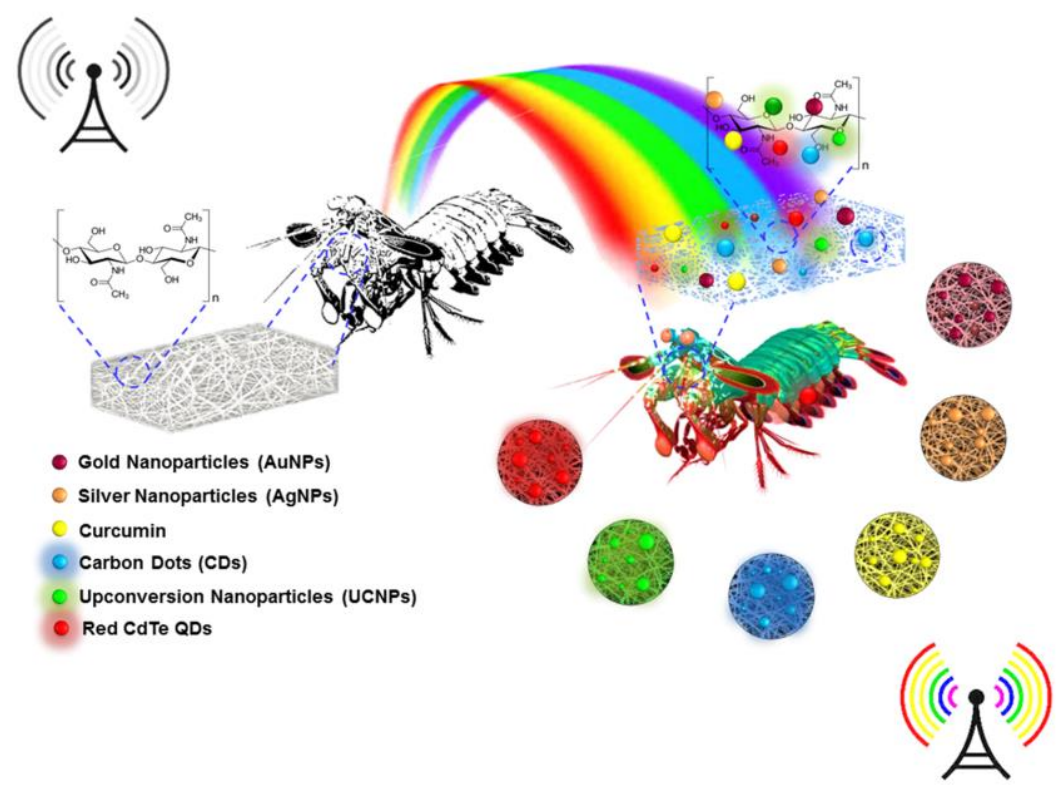

